\title{
4-Aminobenzoic Acid Derivatives: Converting Folate Precursor to Antimicrobial and Cytotoxic Agents
}

\author{
Martin Krátký $1, * \mathbb{( 1 )}$, Klára Konečná ${ }^{2}\left(\mathbb{D}\right.$, Jiř́i Janoušek ${ }^{3}(D)$, Michaela Brablíková ${ }^{4}$, \\ Ondřej Jand'ourek ${ }^{2}$ D, František Trejtnar $^{3}$, Jiřina Stolaříková ${ }^{5}$ and Jarmila Vinšová 1 \\ 1 Department of Organic and Bioorganic Chemistry, Faculty of Pharmacy in Hradec Králové, Charles \\ University, Akademika Heyrovského 1203, 50005 Hradec Králové, Czech Republic; vinsova@faf.cuni.cz \\ 2 Department of Biological and Medical Sciences, Faculty of Pharmacy in Hradec Králové, Charles University, \\ Akademika Heyrovského 1203, 50005 Hradec Králové, Czech Republic; konecna@faf.cuni.cz (K.K.); \\ jando6aa@faf.cuni.cz (O.J.) \\ 3 Department of Pharmacology and Toxicology, Faculty of Pharmacy in Hradec Králové, Charles University, \\ Akademika Heyrovského 1203, 50005 Hradec Králové, Czech Republic; janousj2@faf.cuni.cz (J.J.); \\ trejtnarf@faf.cuni.cz (F.T.) \\ 4 Unipetrol Centre of Research and Education, 43670 Litvínov-Záluží 1, Czech Republic; \\ brablikova.michaela@gmail.com \\ 5 Laboratory for Mycobacterial Diagnostics and Tuberculosis, Regional Institute of Public Health in Ostrava, \\ Partyzánské náměstí 7, 70200 Ostrava, Czech Republic; Jirina.Stolarikova@zuova.cz \\ * Correspondence: martin.kratky@faf.cuni.cz; Tel.: +420-495-067-343; Fax: +420-495-067-166
}

Received: 5 November 2019; Accepted: 17 December 2019; Published: 19 December 2019

check for updates

\begin{abstract}
PABA), an essential nutrient for many human pathogens, but dispensable for humans, and its derivatives have exhibited various biological activities. In this study, we combined two pharmacophores using a molecular hybridization approach: this vitamin-like molecule and various aromatic aldehydes, including salicylaldehydes and 5-nitrofurfural, via imine bond in one-step reaction. Resulting Schiff bases were screened as potential antimicrobial and cytotoxic agents. The simple chemical modification of non-toxic PABA resulted in constitution of antibacterial activity including inhibition of methicillin-resistant Staphylococcus aureus (minimum inhibitory concentrations, MIC, from $15.62 \mu \mathrm{M}$ ), moderate antimycobacterial activity (MIC $\geq 62.5 \mu \mathrm{M}$ ) and potent broad-spectrum antifungal properties (MIC of $\geq 7.81 \mu \mathrm{M}$ ). Some of the Schiff bases also exhibited notable cytotoxicity for cancer HepG2 cell line $\left(\mathrm{IC}_{50} \geq 15.0 \mu \mathrm{M}\right)$. Regarding aldehyde used for the derivatization of PABA, it is possible to tune up the particular activities and obtain derivatives with promising bioactivities.
\end{abstract}

Keywords: 4-aminobenzoic acid; antibacterial activity; antifungal activity; cytotoxicity; Schiff bases; synthesis; vitamin

\section{Introduction}

Para-aminobenzoic acid (4-aminobenzoic acid, $p$-aminobenzoic acid; PABA; 1 ) is a well-known amino acid compound in biochemistry, medicinal chemistry and with many industrial applications.

PABA is synthesized and utilized as a substrate for the synthesis of folic acid in many bacterial species, yeasts and plants, i.e., playing a crucial role in this metabolic pathway. In addition to folate metabolism, PABA was identified as a coenzyme $Q$ precursor and one of the factors required for microbial virulence. From traditional point of view, this compound belongs to the vitamins $B$ group (vitamin $\mathrm{B}_{\mathrm{x}}$ or $\mathrm{B}_{10}$ ). However, PABA is neither essential nor biosynthesized in mammals. It is produced by symbiotic bacteria, especially Escherichia coli and absorbed from dietary intake [1-5]. 
In contrast to its essentiality for some bacteria, when combined with several antibiotics, PABA applies a synergistic antibacterial potency for different bacterial strain including Staphylococcus aureus or Pseudomonas aeruginosa. PABA alone showed a direct antibacterial activity against Listeria monocytogenes, Salmonella enteritidis and E. coli. Low $\mathrm{pH}$ values potentiate this action. Analogous conclusions about adjunctive/synergistic activity were obtained for antivirotics [1-3,6]. It has also exhibited multiple antiviral and antioxidant actions. PABA and its derivatives have been utilized as active constituents in sunscreens to protect against UVB irradiation, i.e., scavengers of reactive oxygen species [1-3]. PABA demonstrated also properties of cytoprotective agents [7] and direct anticoagulants [2]. PABA-based diagnostic tests of gastrointestinal tract state have been developed $[4,8]$. The potassium 4-aminobenzoate is used for the treatment of mainly dermatologic disorders such as scleroderma, dermatomyositis and Peyronie's disease [3]. In agriculture, PABA has displayed the promotion of resistance against plant bacterial and viral pathogens and plant growth modulator properties [4].

Nevertheless, absorption of PABA in the gastrointestinal tract represents an unfavourable feature [3]. The acid is absorbed through a passive process and it undergoes phase II biotransformation. PABA can be metabolized by either $N$-acetylation or in conjugation with glycine in the liver to produce 4-aminohippuric acid. Both these metabolic processes can take place to synthesize 4-acetamidohippuric acid. Then, PABA and its metabolites are almost completely excreted in the urine. PABA is generally considered non-toxic and well-tolerated. Side effects of systemic exposure are predominantly non-specific (nausea and skin rash), although hepatotoxicity may occur [8,9].

As mentioned above, the organisms biosynthesizing PABA obtain it from chorismate by a reaction catalysed by 4-amino-4-deoxychorismate synthase. This enzyme replaces the hydroxyl group at $\mathrm{C} 4$ by an amino group from chorismate, and it removes the pyruvic moiety subsequently to form 4-aminobenzoate [10]. The structure of PABA is highly specific and minimal structural alterations lead to inhibition of enzymes utilizing PABA or involved in subsequent metabolic steps. Illustratively, sulfonamides used for the treatment of many bacterial and protozoal infections act as structural analogues of PABA inhibiting dihydropteroate synthase competitively [11]. A natural metabolite of PABA, 4-acetamidobenzoic acid (acedoben, Figure 1, I), is also able to interfere with the function of dihydropteroate synthase [12]. This molecule is one of the components of immunomodulating and antiviral drug inosine pranobex (Isoprinosine; Figure 1, II) [13]. Antitubercular drug 4-aminosalicylic acid (Figure 1, III) is utilized by dihydropteroate synthase instead of PABA and incorporated into the folate pathway to generate a hydroxyl dihydrofolate antimetabolite, which interferes with dihydrofolate reductase activity leading to the disruption of the biosynthesis of purines and thymidylate [11,14]. The 4-aminoaryl group is required for the biological functions and essentiality of PABA. Its chemical modification prevents its vitamin action and utilization.

In medicinal chemistry, a PABA-building block is used in design of drugs frequently and this scaffold can be found in many drugs with various therapeutic effects (Figure 1). We can distinguish "simple" PABA-derived drugs substituted at carboxyl and/or amino group basically, or more complex molecules with an additional substitution of benzene ring. Focusing on the first group mentioned, the carboxyl can be free, in salt form, or modified through ester or amide bond formation. The amino group is usually free, mono- and di-alkylated or incorporated into amide. For example, PABA-based drugs summarized in Figure 1 involve anticancer and cytotoxic folate antagonists (e.g., aminopterin $I V$, methotrexate $V$ ), alkylating agents, local anaesthetics (benzocaine VI, tetracaine VII, procaine $V I I I$, etc.), antiarrhythmics (procainamide $I X$ ), sunscreens (Padimate-O $X$ ) or anti-inflammatory drugs (balsalazide $X I$ ) [12]. 


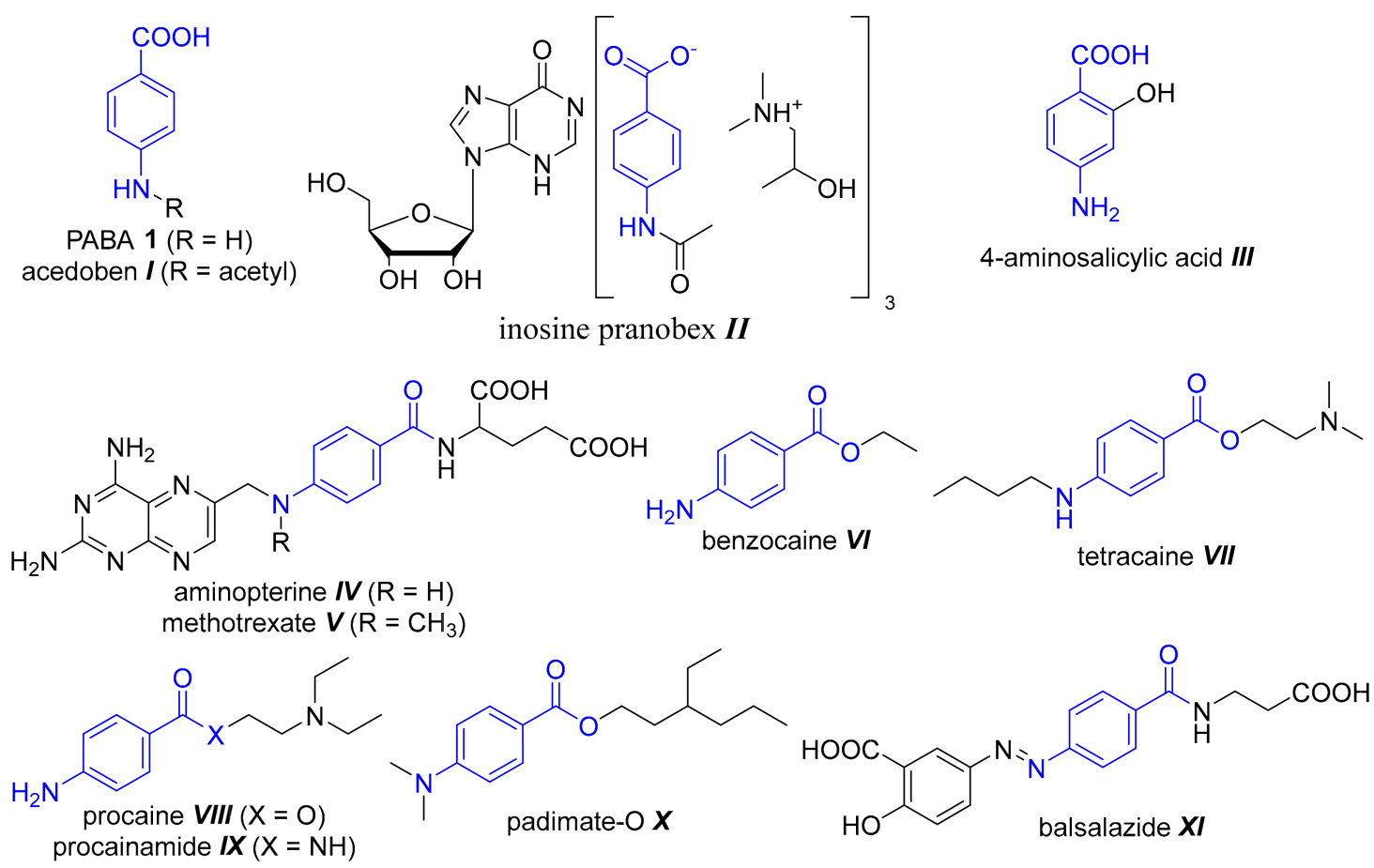

Figure 1. Examples of drugs containing the 4-aminobenzoic acid motif.

PABA hybrid compounds have been reported as potential antimicrobial agents. PABA has been linked with other bioactive scaffolds involving $\mathrm{NH}_{2}$ group via amide bond [15], as a secondary amine [1] or ureido moiety [16]. The derivatives of PABA showed improved radical scavenging properties and also enhanced toxicity for cancer cells [5]. PABA-ambazone salt exhibited improved antibacterial effect when compared to parent drugs [17]. Schiff bases with isatin (Figure 2, XII) exhibited activity against various cancer cell lines [18]. Thus, the chemical modification of PABA provides a viable concept for modulation of its properties to obtain both antimicrobial and cytotoxic compounds.<smiles>[X]c1ccc2c(c1)/C(=N/c1ccc(C(=O)O)cc1)C(=O)N2[R]</smiles>

$\mathrm{R}=\mathrm{H}, \mathrm{CH}_{3}, \mathrm{Bz}$ $\mathrm{X}=\mathrm{H}, \mathrm{F}, \mathrm{Cl}, \mathrm{Br}$ isatin derivatives $\mathbf{X I I}$<smiles>[X]c1ccc(O)c(/C=N/NC(=O)c2ccc(C(F)(F)F)cc2)c1</smiles>

salicylaldehyde-based hydrazones XIII salicylaldehyde-sulfonamide imines XIV

Figure 2. Examples of bioactive PABA (blue) and salicylaldehyde-derived (green) imines and hydrazones.

Our present approach is focused on the synthesis of Schiff bases of PABA and aldehydes, mainly salicylaldehydes, and their evaluation as potential antimicrobial and cytotoxic agents. These fused compounds combine two pharmacophores through the imine bond. Previously, we have applied molecular hybridization approach successfully. Illustratively, salicylaldehyde-based imines and hydrazones derived from known bioactive scaffolds (Figure 2, XIII-XIV) have exhibited a significant antimicrobial activity [19-21]. We have described that a potent antimicrobial activity is associated only with the salicylidene moiety and its isomers are substantially less active [21,22]. That is why we designed predominantly 2-substituted benzylidene scaffold for this study. The modification of the amino group can result in altered vitamin function in susceptible strains and novel derivatives with an 
enhanced lipophilicity may promote limited absorption and intracellular accumulation of PABA via passive diffusion.

\section{Materials and Methods}

\subsection{Chemistry}

\subsubsection{General}

All the reagents and solvents were purchased from Sigma-Aldrich (Darmstadt, Germany) or Penta Chemicals (Prague, Czech Republic) and they were used as received. The reactions and the purity of the products were monitored by thin-layer chromatography (TLC) using a mixture with a ratio of dichloromethane (DCM) to methanol (MeOH) of 4:1 (v/v) as the eluent. TLC plates were coated with 0.2 mm Merck 60 F254 silica gel (Merck Millipore, Darmstadt, Germany) with UV detection (254 nm). The melting points were determined on a Büchi Melting Point B-540 apparatus (BÜCHI, Flawil, Switzerland) using open capillaries. The reported values are uncorrected. Infrared spectra were recorded on a FT-IR spectrometer using ATR-Ge method (Nicolet 6700 FT-IR, Thermo Fisher Scientific, Waltham, MA, USA) in the range of $650-4000 \mathrm{~cm}^{-1}$. The NMR spectra were measured in DMSO- $d_{6}$ at ambient temperature using a Varian V NMR S500 instrument (500 MHz for ${ }^{1} \mathrm{H}$ and $126 \mathrm{MHz}$ for ${ }^{13} \mathrm{C}$; Varian Comp. Palo Alto, CA, USA). The chemical shifts $\delta$ are given in ppm and were referred indirectly to tetramethylsilane via signals of DMSO- $d_{6}$ (2.49 for ${ }^{1} \mathrm{H}$ and 39.7 for ${ }^{13} \mathrm{C}$ spectra). The coupling constants $(J)$ are reported in $\mathrm{Hz}$. Elemental analysis was performed on Vario MICRO Cube Element Analyzer (Elementar Analysensysteme, Hanau, Germany). Both calculated and found values are given as percentages.

\subsubsection{Synthesis of PABA-Derived Schiff Bases 1a-1t}

4-aminobenzoic acid (137.1 mg; $1 \mathrm{mmol}$ ) was dissolved in $7 \mathrm{~mL}$ of $\mathrm{MeOH}$ and then appropriate aldehyde $(1.1 \mathrm{mmol})$ was added in one portion. The reaction mixture was heated under reflux for $3 \mathrm{~h}$, then let stir at room temperature for $12 \mathrm{~h}$. After this time, the reaction mixture was stored at $-20^{\circ} \mathrm{C}$ for $1 \mathrm{~h}$. The resulted precipitate was filtered off and washed by cold diethyl ether (DEE) thoroughly. The products were crystallized from $\mathrm{MeOH}$ to obtain pure Schiff bases if necessary. Alternatively, Schiff bases can be prepared using DEE as a solvent instead of $\mathrm{MeOH}$. In this case, the reaction mixture was stirred for $72 \mathrm{~h}$ at room temperature. The practical yields of both methods were comparable.

Toluene-4-sulfonic acid (as monohydrate; $19.0 \mathrm{mg}$; 0.1 of equivalents) was applied as an acidic catalyst in the synthesis of two products $(\mathbf{1 f}, \mathbf{1 t})$, when the uncatalyzed reaction provided low or almost no yields.

The identity of the known compounds was established using NMR and IR spectroscopy by the comparison with previously reported data. Additionally, their purity was checked by melting points measurement and elemental analysis. The compounds were considered pure if they agree within \pm $0.4 \%$ with theoretical values.

4-[(2-Hydroxybenzylidene)amino]benzoic acid (1a). Yellow solid; yield 92\%; mp 265.5-268 ${ }^{\circ} \mathrm{C}$ (lit. [23] 276-278 $\left.{ }^{\circ} \mathrm{C}\right)$. IR: $1690(\mathrm{COOH}), 1608(-\mathrm{HC}=\mathrm{N}-) \mathrm{cm}^{-1} .{ }^{1} \mathrm{H}-\mathrm{NMR}\left(500 \mathrm{MHz}, \mathrm{DMSO}-d_{6}\right): \delta 12.95(1 \mathrm{H}$, $\mathrm{s}, \mathrm{COOH}), 12.71(1 \mathrm{H}, \mathrm{s}, \mathrm{OH}), 8.99(1 \mathrm{H}, \mathrm{s}, \mathrm{CH}=\mathrm{N}), 8.03-7.99(2 \mathrm{H}, \mathrm{m}, \mathrm{H} 2, \mathrm{H} 6), 7.69(1 \mathrm{H}, \mathrm{dd}, \mathrm{J}=7.6$ $\left.\mathrm{Hz}, 1.8 \mathrm{~Hz}, \mathrm{H6}^{\prime}\right), 7.51-7.42$ (3H, m, H3, H5, H4'), 7.02-6.96 (2H, m, H3' , H5'). ${ }^{13} \mathrm{C}-\mathrm{NMR}(126 \mathrm{MHz}$, DMSO- $\left.d_{6}\right): \delta 167.06,164.99,160.49,152.34,134.02,132.80,130.92,128.11,121.71,121.22,119.50,115.88$. Anal. Calcd. for $\mathrm{C}_{14} \mathrm{H}_{11} \mathrm{NO}_{3}$ (241.25): C, 69.70; H, 4.60; N, 5.81. Found: C, 69.79; H, 4.74; N, 5.70.

4-[(5-Fluoro-2-hydroxybenzylidene)amino]benzoic acid (1b). Orange solid; yield 90\%; mp 277-278.5 ${ }^{\circ} \mathrm{C}$. IR: $1678(\mathrm{COOH}), 1570(-\mathrm{HC}=\mathrm{N}-) \mathrm{cm}^{-1} .{ }^{1} \mathrm{H}-\mathrm{NMR}\left(500 \mathrm{MHz}, \mathrm{DMSO}-d_{6}\right): \delta 12.92(1 \mathrm{H}, \mathrm{s}, \mathrm{COOH})$, $12.28(1 \mathrm{H}, \mathrm{s}, \mathrm{OH}), 8.94(1 \mathrm{H}, \mathrm{s}, \mathrm{CH}=\mathrm{N}), 8.03-7.99(2 \mathrm{H}, \mathrm{m}, \mathrm{H} 2, \mathrm{H} 6), 7.54\left(1 \mathrm{H}, \mathrm{dd}, J=8.9 \mathrm{~Hz}, 3.2 \mathrm{~Hz}, \mathrm{H6}^{\prime}\right)$, 7.48-7.43 (2H, m, H3, H5), 7.30 (ddd, J = 9.1 Hz, 8.3 Hz, 3.2 Hz, H4'), $7.00(1 \mathrm{H}, \mathrm{dd}, J=9.1 \mathrm{~Hz}, 4.5 \mathrm{~Hz}$, H3' $) .{ }^{13} \mathrm{C}-\mathrm{NMR}\left(126 \mathrm{MHz}, \mathrm{DMSO}-d_{6}\right): \delta 167.05,163.30(\mathrm{~d}, J=2.7 \mathrm{~Hz}), 156.64,155.20(\mathrm{~d}, J=234.9 \mathrm{~Hz})$, 
152.46, 130.94, 129.15, 121.70, $120.97(\mathrm{~d}, J=23.5 \mathrm{~Hz}), 119.94(\mathrm{~d}, J=7.5 \mathrm{~Hz}), 118.34(\mathrm{~d}, J=7.6 \mathrm{~Hz}), 116.90$ $(\mathrm{d}, \mathrm{J}=23.4 \mathrm{~Hz})$. Anal. Calcd. for $\mathrm{C}_{14} \mathrm{H}_{10} \mathrm{FNO}_{3}$ (259.24): C, 64.87; H, 3.89; N, 5.40. Found: C, 64.69; $\mathrm{H}$, $3.71 ; \mathrm{N}, 5.64$.

4-[(5-Chloro-2-hydroxybenzylidene)amino]benzoic acid (1c). Yellow solid; yield 91\%; mp 302.5-304 ${ }^{\circ} \mathrm{C}$ (lit. [24] 230-232 $\left.{ }^{\circ} \mathrm{C}\right)$. IR: 1681 (COOH), 1599 (-HC=N-) cm ${ }^{-1}$. ${ }^{1} \mathrm{H}-\mathrm{NMR}\left(500 \mathrm{MHz}, \mathrm{DMSO}-d_{6}\right): \delta$ $12.97(1 \mathrm{H}, \mathrm{s}, \mathrm{COOH}), 12.60(1 \mathrm{H}, \mathrm{s}, \mathrm{OH}), 8.95(1 \mathrm{H}, \mathrm{s}, \mathrm{CH}=\mathrm{N}), 8.04-7.99(2 \mathrm{H}, \mathrm{m}, \mathrm{H} 2, \mathrm{H} 6), 7.78(1 \mathrm{H}, \mathrm{d}$, $\left.J=2.7 \mathrm{~Hz}, \mathrm{H}^{\prime}\right), 7.48-7.43\left(3 \mathrm{H}, \mathrm{m}, \mathrm{H} 3, \mathrm{H} 5, \mathrm{H} 4^{\prime}\right), 7.01\left(1 \mathrm{H}, \mathrm{d}, J=8.8 \mathrm{~Hz}, \mathrm{H} 3^{\prime}\right) .{ }^{13} \mathrm{C}-\mathrm{NMR}(126 \mathrm{MHz}$, DMSO- $\left.d_{6}\right): \delta 167.02,163.24,159.05,152.27,133.42,131.01,130.93,129.21,122.93,121.73,120.89,118.93$. Anal. Calcd. for $\mathrm{C}_{14} \mathrm{H}_{10} \mathrm{ClNO}_{3}$ (275.69): C, 60.99; H, 3.66; N, 5.08. Found: C, 61.11; H, 3.72; N, 5.16.

4-[(5-Bromo-2-hydroxybenzylidene)amino]benzoic acid (1d). Orange solid; yield 87\%; mp 281.5-284 ${ }^{\circ} \mathrm{C}$ (lit. [25] $\left.282{ }^{\circ} \mathrm{C}\right)$. IR: $1681(\mathrm{COOH}), 1596(-\mathrm{HC}=\mathrm{N}-) \mathrm{cm}^{-1} .{ }^{1} \mathrm{H}-\mathrm{NMR}\left(500 \mathrm{MHz}, \mathrm{DMSO}-d_{6}\right): \delta 12.92$ $(1 \mathrm{H}, \mathrm{s}, \mathrm{COOH}), 12.62(1 \mathrm{H}, \mathrm{s}, \mathrm{OH}), 8.94(1 \mathrm{H}, \mathrm{s}, \mathrm{CH}=\mathrm{N}), 8.03-7.99(2 \mathrm{H}, \mathrm{m}, \mathrm{H} 2, \mathrm{H} 6), 7.90(1 \mathrm{H}, \mathrm{d}, \mathrm{J}=2.6 \mathrm{~Hz}$, $\left.\mathrm{H6}^{\prime}\right), 7.57\left(1 \mathrm{H}, \mathrm{dd}, J=8.8 \mathrm{~Hz}, 2.6 \mathrm{~Hz}, \mathrm{H}^{\prime}\right), 7.48-7.44(2 \mathrm{H}, \mathrm{m}, \mathrm{H} 3, \mathrm{H} 5), 6.96\left(1 \mathrm{H}, \mathrm{d}, J=8.8 \mathrm{~Hz}, \mathrm{H3}^{\prime}\right)$. ${ }^{13} \mathrm{C}-\mathrm{NMR}\left(126 \mathrm{MHz}\right.$, DMSO- $\left.d_{6}\right): \delta 167.04,163.18,159.47,152.27,136.20,134.00,130.95,129.22,121.75$, 121.52, 119.36, 110.31. Anal. Calcd. for $\mathrm{C}_{14} \mathrm{H}_{10} \mathrm{BrNO}_{3}$ (320.14): C, 52.52; H, 3.15; N, 4.38. Found: C, $52.25 ; \mathrm{H}, 3.04 ; \mathrm{N}, 4.44$.

4-[(2-Hydroxy-5-iodobenzylidene)amino]benzoic acid (1e). Orange solid; yield 96\%; mp 302-304 ${ }^{\circ} \mathrm{C}$. IR: $1680(\mathrm{COOH}), 1593(-\mathrm{HC}=\mathrm{N}-) \mathrm{cm}^{-1} .{ }^{1} \mathrm{H}-\mathrm{NMR}\left(500 \mathrm{MHz}, \mathrm{DMSO}-d_{6}\right): \delta 12.96(1 \mathrm{H}, \mathrm{s}, \mathrm{COOH}), 12.64$ $(1 \mathrm{H}, \mathrm{s}, \mathrm{OH}), 8.92(1 \mathrm{H}, \mathrm{s}, \mathrm{CH}=\mathrm{N}), 8.04\left(1 \mathrm{H}, \mathrm{d}, J=2.3 \mathrm{~Hz}, \mathrm{H}^{\prime}\right), 8.03-7.99(2 \mathrm{H}, \mathrm{m}, \mathrm{H} 2, \mathrm{H} 6), 7.70(1 \mathrm{H}, \mathrm{dd}, J$ $\left.=8.7 \mathrm{~Hz}, 2.3 \mathrm{~Hz}, \mathrm{H}^{\prime}\right)$, 7.48-7.44 (2H, m, H3, H5), $6.84\left(1 \mathrm{H}, \mathrm{d}, J=8.7 \mathrm{~Hz}, \mathrm{H}^{\prime}\right) .{ }^{13} \mathrm{C}-\mathrm{NMR}(126 \mathrm{MHz}$, DMSO- $d_{6}$ ): $\delta 167.02,163.20,160.00,152.26,141.81,140.04,130.93,129.16,122.12,121.74,119.64,81.08$. Anal. Calcd. for $\mathrm{C}_{14} \mathrm{H}_{10} \mathrm{INO}_{3}$ (367.14): C, 45.80; H, 2.75; N, 3.82. Found: C, 46.02; H, 3.00; N, 3.55.

4-[(2-Hydroxy-5-nitrobenzylidene)amino]benzoic acid (1f). Yellowish orange solid; yield 81\%; $\mathrm{mp}$ 311-313 ${ }^{\circ} \mathrm{C}$ (decomp.). IR: $1693(\mathrm{COOH}), 1597(-\mathrm{HC}=\mathrm{N}-) \mathrm{cm}^{-1} .{ }^{1} \mathrm{H}-\mathrm{NMR}\left(500 \mathrm{MHz}, \mathrm{DMSO}-d_{6}\right): \delta$ $13.78(1 \mathrm{H}, \mathrm{s}, \mathrm{COOH}), 12.87(1 \mathrm{H}, \mathrm{s}, \mathrm{OH}), 9.14(1 \mathrm{H}, \mathrm{s}, \mathrm{CH}=\mathrm{N}), 8.69\left(1 \mathrm{H}, \mathrm{d}, J=2.9 \mathrm{~Hz}, \mathrm{H6} 6^{\prime}\right), 8.28(1 \mathrm{H}, \mathrm{dd}$, $\left.J=9.2 \mathrm{~Hz}, 2.9 \mathrm{~Hz}, \mathrm{H} 4^{\prime}\right), 8.05-8.01(2 \mathrm{H}, \mathrm{m}, \mathrm{H} 2, \mathrm{H} 6), 7.54-7.50(3 \mathrm{H}, \mathrm{m}, \mathrm{H} 3, \mathrm{H} 5), 7.14(1 \mathrm{H}, \mathrm{d}, J=9.2 \mathrm{~Hz}$, $\left.\mathrm{H3}^{\prime}\right) .{ }^{13} \mathrm{C}-\mathrm{NMR}(126 \mathrm{MHz}$, DMSO-d 6 ): $\delta$ 166.95, 166.31, 162.66, 151.24, 139.63, 130.95, 129.56, 128.90, 128.16, 121.74, 119.21, 118.36. Anal. Calcd. for $\mathrm{C}_{14} \mathrm{H}_{10} \mathrm{~N}_{2} \mathrm{O}_{5}$ (286.24): C, 58.75; H, 3.52; N, 9.79. Found: C, 58.86; H, 3.33; N, 10.02 .

4-[(2-Hydroxy-5-methylbenzylidene)amino]benzoic acid (1g). Yellow solid; yield 92\%; mp 271-273 ${ }^{\circ} \mathrm{C}$ (lit. [26] $\left.258{ }^{\circ} \mathrm{C}\right)$. IR: $1681(\mathrm{COOH}), 1574(-\mathrm{HC}=\mathrm{N}-) \mathrm{cm}^{-1} .{ }^{1} \mathrm{H}-\mathrm{NMR}\left(500 \mathrm{MHz}, \mathrm{DMSO}-d_{6}\right): \delta 12.94$ $(1 \mathrm{H}, \mathrm{s}, \mathrm{COOH}), 12.41(1 \mathrm{H}, \mathrm{s}, \mathrm{OH}), 8.91(1 \mathrm{H}, \mathrm{s}, \mathrm{CH}=\mathrm{N}), 8.03-7.99(2 \mathrm{H}, \mathrm{m}, \mathrm{H} 2, \mathrm{H} 6), 7.50-7.43$ (3H, m, H3, H5, H6' $), 7.25\left(1 \mathrm{H}, \mathrm{dd}, J=8.4 \mathrm{~Hz}, 2.3 \mathrm{~Hz}, \mathrm{H}^{\prime}\right), 6.88\left(1 \mathrm{H}, \mathrm{d}, J=8.3 \mathrm{~Hz}, \mathrm{H}^{\prime}\right), 2.27\left(3 \mathrm{H}_{,}, \mathrm{s}, \mathrm{CH}_{3}\right)$. ${ }^{13} \mathrm{C}-\mathrm{NMR}\left(126 \mathrm{MHz}\right.$, DMSO-d $\left.d_{6}\right): \delta 167.05,164.74,158.33,152.52,134.78,132.42,130.90,128.87,128.04$, 121.63, 119.18, 116.72, 20.09. Anal. Calcd. for $\mathrm{C}_{15} \mathrm{H}_{13} \mathrm{NO}_{3}$ (255.27): C, 70.58; H, 5.13; N, 5.49. Found: C, $70.81 ; \mathrm{H}, 5.01 ; \mathrm{N}, 5.57$.

4-[(2-Hydroxy-5-methoxybenzylidene)amino]benzoic acid (1h) [27]. Brownish yellow solid; yield 92\%; mp 247.5-249.5 ${ }^{\circ} \mathrm{C}$ (decomp.). IR: 1677 (COOH), 1569 (-HC=N-) cm ${ }^{-1}$. ${ }^{1} \mathrm{H}-\mathrm{NMR}\left(500 \mathrm{MHz}, \mathrm{DMSO}-d_{6}\right)$ : $\delta 12.93(1 \mathrm{H}, \mathrm{s}, \mathrm{COOH}), 12.04(1 \mathrm{H}, \mathrm{s}, \mathrm{OH}), 8.94(1 \mathrm{H}, \mathrm{s}, \mathrm{CH}=\mathrm{N}), 8.03-7.99(2 \mathrm{H}, \mathrm{m}, \mathrm{H} 2, \mathrm{H} 6), 7.46-7.43(2 \mathrm{H}$, m, H3, H5), $7.28\left(1 \mathrm{H}, \mathrm{d}, J=3.2 \mathrm{~Hz}, \mathrm{H}^{\prime}\right), 7.06\left(1 \mathrm{H}, \mathrm{dd}, J=9.0 \mathrm{~Hz}, 3.2 \mathrm{~Hz}, \mathrm{H} 4^{\prime}\right), 6.92(1 \mathrm{H}, \mathrm{d}, J=8.9 \mathrm{~Hz}$, $\left.\mathrm{H}^{\prime}\right), 3.75\left(3 \mathrm{H}, \mathrm{s}, \mathrm{OCH}_{3}\right) .{ }^{13} \mathrm{C}-\mathrm{NMR}\left(126 \mathrm{MHz}, \mathrm{DMSO}-d_{6}\right): \delta 167.06,164.31,154.58,152.70,152.15$, $130.92,128.89,121.63,121.36,119.44,117.81,115.01,55.73$. Anal. Calcd. for $\mathrm{C}_{15} \mathrm{H}_{13} \mathrm{NO}_{4}$ (271.27): C, 66.41; H, 4.83; N, 5.16. Found: C, 66.35; H, 4.69; N, 5.01.

4-[(2,5-Dihydroxybenzylidene)amino]benzoic acid (1i) [28]. Orange solid; yield 81\%; mp 278-280 ${ }^{\circ} \mathrm{C}$ (decomp.). IR: $1679(\mathrm{COOH}), 1574(-\mathrm{HC}=\mathrm{N}-) \mathrm{cm}^{-1} .{ }^{1} \mathrm{H}-\mathrm{NMR}\left(500 \mathrm{MHz}, \mathrm{DMSO}-d_{6}\right): \delta 12.89(1 \mathrm{H}$, s, $\mathrm{COOH}), 11.87(1 \mathrm{H}, \mathrm{s}, 2-\mathrm{OH}), 9.13(1 \mathrm{H}, \mathrm{s}, 5-\mathrm{OH}), 8.87(1 \mathrm{H}, \mathrm{s}, \mathrm{CH}=\mathrm{N}), 8.02-7.98(2 \mathrm{H}, \mathrm{m}, \mathrm{H} 2, \mathrm{H} 6)$, 7.46-7.43 (2H, m, H3, H5), $7.08\left(1 \mathrm{H}, \mathrm{d}, J=3.0 \mathrm{~Hz}, \mathrm{H6} 6^{\prime}\right), 6.89\left(1 \mathrm{H}, \mathrm{dd}, J=8.8 \mathrm{~Hz}, 3.0 \mathrm{~Hz}, \mathrm{H} 4^{\prime}\right), 6.81(1 \mathrm{H}$, $\left.\mathrm{d}, J=8.8 \mathrm{~Hz}, \mathrm{H3}^{\prime}\right) .{ }^{13} \mathrm{C}-\mathrm{NMR}\left(126 \mathrm{MHz}, \mathrm{DMSO}-d_{6}\right): \delta 167.08,164.50,153.35,152.78,149.92,130.88$, $128.78,121.95,121.66,119.54,117.52,116.89$. Anal. Calcd. for $\mathrm{C}_{14} \mathrm{H}_{11} \mathrm{NO}_{4}$ (257.07): C, 65.37; $\mathrm{H}, 4.31 ; \mathrm{N}$, 5.45. Found: C, 65.55; H, 4.40; N, 5.62. 
4-\{[5-(Tert-butyl)-2-hydroxybenzylidene]amino\}benzoic acid (1j). Orange solid; yield 85\%; mp 270-272 ${ }^{\circ} \mathrm{C}$. IR: $1682(\mathrm{COOH}), 1569(-\mathrm{HC}=\mathrm{N}-) \mathrm{cm}^{-1} .{ }^{1} \mathrm{H}-\mathrm{NMR}\left(500 \mathrm{MHz}, \mathrm{DMSO}-d_{6}\right): \delta 12.94(1 \mathrm{H}, \mathrm{s}, \mathrm{COOH})$, $12.51(1 \mathrm{H}, \mathrm{s}, \mathrm{OH}), 8.99(1 \mathrm{H}, \mathrm{s}, \mathrm{CH}=\mathrm{N}), 8.03-7.99(2 \mathrm{H}, \mathrm{m}, \mathrm{H} 2, \mathrm{H} 6), 7.70\left(1 \mathrm{H}, \mathrm{d}, J=2.6 \mathrm{~Hz}, \mathrm{H} 6^{\prime}\right), 7.50-7.46$ $\left(3 \mathrm{H}, \mathrm{m}, \mathrm{H} 3, \mathrm{H} 5, \mathrm{H} 4^{\prime}\right), 6.92\left(1 \mathrm{H}, \mathrm{d}, J=8.7 \mathrm{~Hz}, \mathrm{H} 3^{\prime}\right), 1.28\left(9 \mathrm{H}, \mathrm{s}, \mathrm{CH}_{3}\right) .{ }^{13} \mathrm{C}-\mathrm{NMR}\left(126 \mathrm{MHz}, \mathrm{DMSO}-d_{6}\right): \delta$ 167.08, 165.40, 158.35, 152.52, 141.66, 131.38, 130.92, 129.04, 128.86, 121.68, 118.75, 116.51, 34.01, 31.35. Anal. Calcd. for $\mathrm{C}_{18} \mathrm{H}_{19} \mathrm{NO}_{3}$ (297.35): C, 72.71; H, 6.44; N, 4.71. Found: C, 72.57; H, 6.58; N, 4.50.

4-[(2-Chloro-6-hydroxybenzylidene)amino]benzoic acid (1k). Orange solid; yield 94\%; mp 279-281 ${ }^{\circ} \mathrm{C}$. IR: $1681(\mathrm{COOH}), 1596(-\mathrm{HC}=\mathrm{N}-) \mathrm{cm}^{-1} .{ }^{1} \mathrm{H}-\mathrm{NMR}\left(500 \mathrm{MHz}, \mathrm{DMSO}-d_{6}\right): \delta 14.02(1 \mathrm{H}, \mathrm{s}, \mathrm{COOH})$, $13.03(1 \mathrm{H}, \mathrm{s}, \mathrm{OH}), 9.17(1 \mathrm{H}, \mathrm{s}, \mathrm{CH}=\mathrm{N}), 8.05-8.01(2 \mathrm{H}, \mathrm{m}, \mathrm{H} 2, \mathrm{H} 6), 7.57-7.53(2 \mathrm{H}, \mathrm{m}, \mathrm{H} 3, \mathrm{H} 5), 7.45(1 \mathrm{H}, \mathrm{t}$, $\left.J=8.2 \mathrm{~Hz}, \mathrm{H} 4^{\prime}\right), 7.07\left(1 \mathrm{H}, \mathrm{dd}, J=7.9 \mathrm{~Hz}, 1.1 \mathrm{~Hz}, \mathrm{H}^{\prime}\right), 6.98\left(1 \mathrm{H}, \mathrm{dd}, J=8.4 \mathrm{~Hz}, 1.0 \mathrm{~Hz}, \mathrm{H} 5^{\prime}\right) .{ }^{13} \mathrm{C}-\mathrm{NMR}$ $(126 \mathrm{MHz}$, DMSO-d 6 ): $\delta 166.95,162.79,161.81,151.04,135.83,134.98,130.99,129.65,121.93,120.46$, 116.83, 115.66. Anal. Calcd. for $\mathrm{C}_{14} \mathrm{H}_{10} \mathrm{ClNO}_{3}$ (275.69): C, 60.99; H, 3.66; N, 5.08. Found: C, 60.84; $\mathrm{H}$, $3.74 ; \mathrm{N}, 5.01$.

4-[(4-Chloro-2-hydroxybenzylidene)amino]benzoic acid (11). Yellowish orange solid; yield 90\%; mp 304-307 ${ }^{\circ} \mathrm{C}$ (decomp.). IR: $1674(\mathrm{COOH}), 1596(-\mathrm{HC}=\mathrm{N}-) \mathrm{cm}^{-1} .{ }^{1} \mathrm{H}-\mathrm{NMR}\left(500 \mathrm{MHz}, \mathrm{DMSO}-d_{6}\right): \delta 13.05$ $(1 \mathrm{H}, \mathrm{s}, \mathrm{COOH}), 12.72(1 \mathrm{H}, \mathrm{s}, \mathrm{OH}), 8.99(1 \mathrm{H}, \mathrm{s}, \mathrm{CH}=\mathrm{N}), 8.04-7.99(2 \mathrm{H}, \mathrm{m}, \mathrm{H} 2, \mathrm{H} 6), 7.72(1 \mathrm{H}, \mathrm{d}, J=8.1 \mathrm{~Hz}$, $\left.\mathrm{H6}^{\prime}\right)$, 7.48-7.43 (2H, m, H3, H5), 7.07-7.03 (2H, m, H3' $\left.{ }^{\prime} \mathrm{H}^{\prime}\right) .{ }^{13} \mathrm{C}-\mathrm{NMR}\left(126 \mathrm{MHz}, \mathrm{DMSO}-d_{6}\right): \delta 167.01$, 163.73, 161.30, 152.01, 138.18, 133.89, 130.90, 129.13, 121.70, 119.79, 118.64, 116.76. Anal. Calcd. for $\mathrm{C}_{14} \mathrm{H}_{10} \mathrm{ClNO}_{3}$ (275.69): C, 60.99; H, 3.66; N, 5.08. Found: $\mathrm{C}, 60.77 ; \mathrm{H}, 3.65 ; \mathrm{N}, 5.20$.

4-[(3-Chloro-2-hydroxybenzylidene)amino]benzoic acid (1m). Yellow solid; yield 85\%; mp 296-298 ${ }^{\circ} \mathrm{C}$. IR: $1689(\mathrm{COOH}), 1596(-\mathrm{CH}=\mathrm{N}-) \mathrm{cm}^{-1} .{ }^{1} \mathrm{H}-\mathrm{NMR}\left(500 \mathrm{MHz}, \mathrm{DMSO}-d_{6}\right): \delta 13.99(1 \mathrm{H}, \mathrm{s}, \mathrm{COOH}), 13.01$ $(1 \mathrm{H}, \mathrm{s}, \mathrm{OH}), 9.08(1 \mathrm{H}, \mathrm{s}, \mathrm{CH}=\mathrm{N}), 8.05-8.01(2 \mathrm{H}, \mathrm{m}, \mathrm{H} 2, \mathrm{H} 6), 7.65\left(1 \mathrm{H}, \mathrm{dd}, J=7.8 \mathrm{~Hz}, 1.6 \mathrm{~Hz}, \mathrm{H6}{ }^{\prime}\right), 7.61$ $\left(1 \mathrm{H}, \mathrm{dd}, J=7.9 \mathrm{~Hz}, 1.5 \mathrm{~Hz}, \mathrm{H} 4^{\prime}\right), 7.57-7.54(2 \mathrm{H}, \mathrm{m}, \mathrm{H} 3, \mathrm{H} 5), 7.01\left(1 \mathrm{H}, \mathrm{t}, J=7.8 \mathrm{~Hz}, \mathrm{H} 5^{\prime}\right) .{ }^{13} \mathrm{C}-\mathrm{NMR}(126$ MHz, DMSO- $d_{6}$ ): $\delta 166.95,165.17,156.69,150.75,133.77,132.22,130.92,129.52,121.87,120.56,120.19$, 119.85. Anal. Calcd. for $\mathrm{C}_{14} \mathrm{H}_{10} \mathrm{ClNO}_{3}$ (275.69): C, 60.99; H, 3.66; N, 5.08. Found: C, 61.09; $\mathrm{H}, 3.79 ; \mathrm{N}$, 5.30 .

4-[(3,5-Dichloro-2-hydroxybenzylidene)amino]benzoic acid (1n). Orange solid; yield 89\%; mp 317.5-319 ${ }^{\circ} \mathrm{C}$ (lit. [29] $\left.300{ }^{\circ} \mathrm{C}\right)$. IR: 1689 (COOH), $1597(-\mathrm{CH}=\mathrm{N}-) \mathrm{cm}^{-1} .{ }^{1} \mathrm{H}-\mathrm{NMR}\left(500 \mathrm{MHz}, \mathrm{DMSO}-d_{6}\right): \delta 14.02$ $(1 \mathrm{H}, \mathrm{s}, \mathrm{COOH}), 13.03(1 \mathrm{H}, \mathrm{s}, \mathrm{OH}), 9.04(1 \mathrm{H}, \mathrm{s}, \mathrm{CH}=\mathrm{N}), 8.05-8.01(2 \mathrm{H}, \mathrm{m}, \mathrm{H} 2, \mathrm{H} 6), 7.75-7.73(2 \mathrm{H}, \mathrm{m}$, $\left.\mathrm{H} 4^{\prime}, \mathrm{H}^{\prime}\right), 7.56-7.53$ (2H, m, H3, H5). ${ }^{13} \mathrm{C}-\mathrm{NMR}\left(126 \mathrm{MHz}, \mathrm{DMSO}-d_{6}\right): \delta 166.90,164.07,155.94,150.30$, 132.83, 131.06, 130.96, 129.83, 122.47, 121.93, 121.88, 120.66. Anal. Calcd. for $\mathrm{C}_{14} \mathrm{H}_{9} \mathrm{Cl}_{2} \mathrm{NO}_{3}$ (309.00): $\mathrm{C}$, 54.22; H, 2.93; N, 4.52. Found: C, 54.10; H, 3.11; N, 4.36.

4-[(3-Bromo-5-chloro-2-hydroxybenzylidene)amino]benzoic acid (1o). Orangish solid; yield 92\%; $\mathrm{mp}>$ $300{ }^{\circ} \mathrm{C}$ (decomp.). IR: 1685 (COOH), $1595(-\mathrm{CH}=\mathrm{N}-) \mathrm{cm}^{-1} .{ }^{1} \mathrm{H}-\mathrm{NMR}\left(500 \mathrm{MHz}, \mathrm{DMSO}-d_{6}\right): \delta 14.05(1 \mathrm{H}$, s, $\mathrm{COOH}), 13.03(1 \mathrm{H}, \mathrm{s}, \mathrm{OH}), 9.02(1 \mathrm{H}, \mathrm{s}, \mathrm{CH}=\mathrm{N}), 8.05-8.02(2 \mathrm{H}, \mathrm{m}, \mathrm{H} 2, \mathrm{H} 6), 7.86(1 \mathrm{H}, \mathrm{d}, J=2.5 \mathrm{~Hz}$, $\left.\mathrm{H}^{\prime}\right), 7.77\left(1 \mathrm{H}, \mathrm{d}, J=2.6 \mathrm{~Hz}, \mathrm{H6} 6^{\prime}\right), 7.57-7.53(2 \mathrm{H}, \mathrm{m}, \mathrm{H} 3, \mathrm{H} 5) .{ }^{13} \mathrm{C}-\mathrm{NMR}\left(126 \mathrm{MHz}, \mathrm{DMSO}-d_{6}\right): \delta 166.88$, 164.08, 156.90, 150.16, 135.60, 131.76, 130.95, 129.82, 122.84, 121.90, 120.38, 111.36. Anal. Calcd. for $\mathrm{C}_{14} \mathrm{H}_{9} \mathrm{BrClNO}_{3}$ (352.95): C, 47.42; H, 2.56; N, 3.95. Found: C, 47.55; H, 2.70; N, 4.13.

4-[(5-Chloro-2-hydroxy-3-iodobenzylidene)amino]benzoic acid (1p). Reddish orange solid; yield 84\%; mp $290{ }^{\circ} \mathrm{C}$ (decomp). IR: $1671(\mathrm{COOH}), 1589(-\mathrm{CH}=\mathrm{N}-) \mathrm{cm}^{-1} .{ }^{1} \mathrm{H}-\mathrm{NMR}\left(500 \mathrm{MHz}, \mathrm{DMSO}-d_{6}\right): \delta 14.04$ $(1 \mathrm{H}, \mathrm{s}, \mathrm{COOH}), 13.03(1 \mathrm{H}, \mathrm{s}, \mathrm{OH}), 8.96(1 \mathrm{H}, \mathrm{s}, \mathrm{CH}=\mathrm{N}), 8.05-8.02(2 \mathrm{H}, \mathrm{m}, \mathrm{H} 2, \mathrm{H} 6), 7.97(1 \mathrm{H}, \mathrm{d}, J=2.6$ $\left.\mathrm{Hz}, \mathrm{H} 4^{\prime}\right), 7.78\left(1 \mathrm{H}, \mathrm{d}, J=2.6 \mathrm{~Hz}, \mathrm{H} 6^{\prime}\right), 7.58-7.53(2 \mathrm{H}, \mathrm{m}, \mathrm{H} 3, \mathrm{H} 5) .{ }^{13} \mathrm{C}-\mathrm{NMR}\left(126 \mathrm{MHz}, \mathrm{DMSO}-d_{6}\right): \delta$ 166.90, 164.09, 159.36, 150.17, 141.24, 132.50, 130.96, 129.77, 123.32, 121.94, 119.23, 87.53. Anal. Calcd. for $\mathrm{C}_{14} \mathrm{H}_{9} \mathrm{ClINO}_{3}$ (400.93): C, 41.87; H, 2.26; N, 3.49. Found: $\mathrm{C}, 42.11 ; \mathrm{H}, 2.39 ; \mathrm{N}, 3.31$.

4-[(2-Hydroxy-3,5-diiodobenzylidene)amino]benzoic acid (1q). Red solid; yield 95\%; mp 289-291 ${ }^{\circ} \mathrm{C}$ (decomp.). IR: $1667(\mathrm{COOH}), 1583(-\mathrm{CH}=\mathrm{N}-) \mathrm{cm}^{-1} .{ }^{1} \mathrm{H}-\mathrm{NMR}\left(500 \mathrm{MHz}, \mathrm{DMSO}-d_{6}\right): \delta 14.08(1 \mathrm{H}, \mathrm{s}$, $\mathrm{COOH}), 13.02(1 \mathrm{H}, \mathrm{s}, \mathrm{OH}), 8.94(1 \mathrm{H}, \mathrm{s}, \mathrm{CH}=\mathrm{N}), 8.16\left(1 \mathrm{H}, \mathrm{d}, J=2.1 \mathrm{~Hz}, \mathrm{H} 4^{\prime}\right), 8.05-8.00(3 \mathrm{H}, \mathrm{m}, \mathrm{H} 2$, H6, H6 $), 7.57-7.53$ (2H, m, H3, H5). ${ }^{13}$ C-NMR (126 MHz, DMSO-d 6 ): $\delta 166.90,163.95,160.22,150.14$, 148.93, 141.47, 130.95, 129.72, 121.91, 120.77, 88.51, 81.51. Anal. Calcd. for $\mathrm{C}_{14} \mathrm{H}_{9} \mathrm{I}_{2} \mathrm{NO}_{3}$ (492.87): C, $34.11 ; \mathrm{H}, 1.84 ; \mathrm{N}, 2.84$. Found: C, 33.87; H, 2.00; N, 2.87. 
4-\{[(5-Nitrofuran-2-yl)methylene]amino\}benzoic acid (1r). Yellow solid; yield $80 \%$; mp 233-235 ${ }^{\circ} \mathrm{C}$ (decomp.) (lit. [30] $234{ }^{\circ} \mathrm{C}$ decomp.). IR: 1673 (COOH), 1591 (-CH=N-) cm ${ }^{-1} .{ }^{1} \mathrm{H}-\mathrm{NMR}(500 \mathrm{MHz}$, DMSO- $\left.d_{6}\right): \delta 12.95(1 \mathrm{H}, \mathrm{s}, \mathrm{COOH}), 8.64(1 \mathrm{H}, \mathrm{s}, \mathrm{CH}=\mathrm{N}), 8.03-7.98(2 \mathrm{H}, \mathrm{m}, \mathrm{H} 2, \mathrm{H} 6), 7.83(1 \mathrm{H}, \mathrm{d}, J=3.9$ $\mathrm{Hz}$, furan $\left.\mathrm{H} 4^{\prime}\right), 7.48\left(1 \mathrm{H}, \mathrm{d}, J=3.9 \mathrm{~Hz}\right.$, furan $\left.\mathrm{H} 3^{\prime}\right), 7.43-7.39(2 \mathrm{H}, \mathrm{m}, \mathrm{H} 3, \mathrm{H} 5) .{ }^{13} \mathrm{C}-\mathrm{NMR}(126 \mathrm{MHz}$, DMSO- $\left.d_{6}\right): \delta 167.00,153.92,152.74,152.47,149.99,130.84,129.37,121.53,119.15,114.20$. Anal. Calcd. for $\mathrm{C}_{12} \mathrm{H}_{8} \mathrm{~N}_{2} \mathrm{O}_{5}$ (260.04): C, 55.39; H, 3.10; N, 10.77. Found: $\mathrm{C}, 55.60 ; \mathrm{H}, 2.94 ; \mathrm{N}, 10.89$.

4-[(2-Methoxybenzylidene)amino]benzoic acid (1s). White solid; yield 95\%; mp 231.5-234 ${ }^{\circ} \mathrm{C}$ (lit. [24] 220-221 $\left.{ }^{\circ} \mathrm{C}\right)$. IR: $1683(\mathrm{COOH}), 1593(-\mathrm{HC}=\mathrm{N}-) \mathrm{cm}^{-1} .{ }^{1} \mathrm{H}-\mathrm{NMR}\left(500 \mathrm{MHz}, \mathrm{DMSO}-d_{6}\right): \delta 12.85(1 \mathrm{H}, \mathrm{s}$, $\mathrm{COOH}), 8.84(1 \mathrm{H}, \mathrm{s}, \mathrm{CH}=\mathrm{N}), 8.02\left(1 \mathrm{H}, \mathrm{dd}, J=7.7 \mathrm{~Hz}, 1.8 \mathrm{~Hz}, \mathrm{H6} 6^{\prime}\right), 7.99-7.95(2 \mathrm{H}, \mathrm{m}, \mathrm{H} 2, \mathrm{H} 6), 7.54(1 \mathrm{H}$, ddd, $\left.J=8.8 \mathrm{~Hz}, 7.2 \mathrm{~Hz}, 1.8 \mathrm{~Hz}, \mathrm{H} 4^{\prime}\right), 7.28-7.23(2 \mathrm{H}, \mathrm{m}, \mathrm{H} 3, \mathrm{H} 5), 7.17\left(1 \mathrm{H}, \mathrm{d}, J=8.4 \mathrm{~Hz}, \mathrm{H} 3^{\prime}\right), 7.07(1 \mathrm{H}, \mathrm{t}$, $\left.J=7.5 \mathrm{~Hz}, \mathrm{H} 5^{\prime}\right) .{ }^{13} \mathrm{C}-\mathrm{NMR}\left(126 \mathrm{MHz}\right.$, DMSO- $\left.d_{6}\right): \delta 167.21,159.72,157.26,156.24,133.92,130.84,127.99$, 127.21, 123.68, 121.09, 120.91, 112.30, 56.01. Anal. Calcd. for $\mathrm{C}_{15} \mathrm{H}_{13} \mathrm{NO}_{3}$ (255.09): C, 70.58; $\mathrm{H}, 5.13 ; \mathrm{N}$, 5.49. Found: C, 70.44; H, 5.02; N, 5.60 .

4-[(4-Hydroxybenzylidene)amino]benzoic acid (1t). Yellowish brown solid; yield 80\%; mp 251-253 ${ }^{\circ} \mathrm{C}$ (lit. [31] 254-255 ${ }^{\circ} \mathrm{C}$ ). IR: $1681(\mathrm{COOH}), 1599(-\mathrm{HC}=\mathrm{N}-) \mathrm{cm}^{-1} .{ }^{1} \mathrm{H}-\mathrm{NMR}\left(500 \mathrm{MHz}, \mathrm{DMSO}-d_{6}\right): \delta$ $10.45(1 \mathrm{H}, \mathrm{s}, \mathrm{COOH}), 9.79(1 \mathrm{H}, \mathrm{s}, \mathrm{OH}), 8.65(1 \mathrm{H}, \mathrm{s}, \mathrm{CH}=\mathrm{N}), 8.00-7.97(2 \mathrm{H}, \mathrm{m}, \mathrm{H} 2, \mathrm{H} 6), 7.79-7.74(2 \mathrm{H}, \mathrm{m}$, $\left.\mathrm{H} 2^{\prime}, \mathrm{H6}^{\prime}\right), 7.38-7.34(2 \mathrm{H}, \mathrm{m}, \mathrm{H} 3, \mathrm{H} 5) .6 .96-6.93\left(2 \mathrm{H}, \mathrm{m}, \mathrm{H} 3^{\prime}, \mathrm{H}^{\prime}\right) .{ }^{13} \mathrm{C}-\mathrm{NMR}\left(126 \mathrm{MHz}, \mathrm{DMSO}-d_{6}\right): \delta$ 167.59, 162.02, 161.41, 156.39, 131.39, 130.86, 128.03, 127.65, 121.12, 116.27. Anal. Calcd. for $\mathrm{C}_{14} \mathrm{H}_{11} \mathrm{NO}_{3}$ (241.25): C, 69.70; H, 4.60; N, 5.81. Found: C, 69.66; H, 4.51; N, 5.97.

\subsubsection{Synthesis of $1 \mathbf{u}$}

4-aminobenzoic acid (137.1 mg; $1 \mathrm{mmol}$ ) was dissolved in $7 \mathrm{~mL}$ of $\mathrm{MeOH}$, followed by addition of salicylaldehyde (117 $\mu \mathrm{L} ; 1.1 \mathrm{mmol})$, sodium cyanoborohydride $(62.8 \mathrm{mg} ; 1 \mathrm{mmol})$ and finally glacial acetic acid $(100 \mu \mathrm{L})$. The mixture was stirred for $24 \mathrm{~h}$ at room temperature. After this time, the reaction mixture was diluted with distilled water and stirred for additional $1 \mathrm{~h}$. The resulted precipitate was filtered off and washed by water. The product was crystallized from ethyl acetate/ $n$-hexane to obtain pure $1 \mathbf{u}$.

4-[(2-Hydroxybenzyl)amino]benzoic acid (1u). Greyish solid; yield 74\%; mp 198-199.5 ${ }^{\circ} \mathrm{C}$ (lit. [32] 203-204 $\left.{ }^{\circ} \mathrm{C}\right)$. IR: 3277, $1670(\mathrm{COOH}) \mathrm{cm}^{-1} .{ }^{1} \mathrm{H}-\mathrm{NMR}\left(500 \mathrm{MHz}, \mathrm{DMSO}-d_{6}\right): \delta 11.95(1 \mathrm{H}, \mathrm{s}, \mathrm{COOH}), 9.56$ $(1 \mathrm{H}, \mathrm{s}, \mathrm{OH}), 7.64(2 \mathrm{H}, \mathrm{d}, J=8.7 \mathrm{~Hz}, \mathrm{H} 2, \mathrm{H} 6), 7.14\left(1 \mathrm{H}, \mathrm{dd}, J=7.6 \mathrm{~Hz}, 2.7 \mathrm{~Hz}, \mathrm{H6} 6^{\prime}\right), 7.05(1 \mathrm{H}, \mathrm{td}, J=7.6$ $\left.\mathrm{Hz}, 1.7 \mathrm{~Hz}, \mathrm{H} 4^{\prime}\right), 6.85-6.79\left(2 \mathrm{H}, \mathrm{m}, \mathrm{NH}, \mathrm{H3} 3^{\prime}\right), 6.73\left(1 \mathrm{H}, \mathrm{td}, J=7.4 \mathrm{~Hz}, 1.1 \mathrm{~Hz}, \mathrm{H} 5^{\prime}\right), 6.60-6.56(2 \mathrm{H}, \mathrm{m}, \mathrm{H} 3$, $\mathrm{H} 5), 4.24\left(2 \mathrm{H}, \mathrm{d}, J=5.8 \mathrm{~Hz}, \mathrm{CH}_{2}\right) .{ }^{13} \mathrm{C}-\mathrm{NMR}\left(126 \mathrm{MHz}, \mathrm{DMSO}-d_{6}\right): \delta 167.68,155.21,152.80,131.26$, 128.39, 127.93, 125.09, 119.00, 117.06, 115.11, 111.14, 41.03. Anal. Calcd. for $\mathrm{C}_{14} \mathrm{H}_{13} \mathrm{NO}_{3}$ (243.09): $\mathrm{C}$, 69.12; H, 5.39; N, 5.76. Found: C, 69.21; H, 5.51; N, 5.49.

\subsection{Pharmacology}

\subsubsection{Antibacterial Activity}

For the purpose of screening for antibacterial activity of the derivatives four Gram-positive and four Gram-negative strains of clinical importance were used, namely: Staphylococcus aureus ATCC 29213, CCM 4223, methicillin-resistant Staphylococcus aureus ATCC 43300, CCM 4750 (MRSA), Staphylococcus epidermidis H 6966/08 (clinical isolate), Enterococcus faecalis ATCC 29212, CCM 4224; Escherichia coli ATCC 25922, CCM 3954, Klebsiella pneumoniae D 11750/08 (clinical isolate), extended-spectrum $\beta$-lactamase positive Klebsiella pneumoniae J 14368/08 (clinical isolate), and Pseudomonas aeruginosa ATCC 27853, CCM 3955. These strains were obtained from the Czech Collection of Microorganisms (CCM, Brno, Czech Republic) or they are clinical isolates from the Department of Clinical Microbiology, University Hospital in Hradec Králové, Czech Republic.

The microdilution broth method was performed according to EUCAST (The European Committee on Antimicrobial Susceptibility Testing) instructions with slight modifications [33]. Briefly, the cultivation was done in Cation-adjusted Mueller-Hinton broth (CAMHB, M-H 2 Broth, Sigma-Aldrich) 
at $35 \pm 2{ }^{\circ} \mathrm{C}$. Tested compounds were dissolved in DMSO (Sigma-Aldrich, St. Louis, MO, USA) to produce stock solutions. The final concentration of DMSO in the testing medium did not exceed $1 \%$ $(v / v)$ of the total solution composition and did not affect the growth of bacteria. Positive (microbe solely), negative (cultivation medium and DMSO) controls and internal quality standards were involved in each assay. Antibacterial activity is expressed as minimum inhibitory concentration (MIC, reported in $\mu \mathrm{M}$ ) after 24 and $48 \mathrm{~h}$ of static incubation in the dark and humidified atmosphere at $35 \pm 2{ }^{\circ} \mathrm{C}$. The experiments were performed in duplicates. For the results to be valid, the difference in MIC determined from two parallel measurements must not be greater than one step on the dilution scale. The results were analysed by visual inspection.

Parent PABA as well as topical antibiotics bacitracin (active especially against Gram-positive species including MRSA) and neomycin (an aminoglycoside active predominantly against Gram-negative strains) were involved as the reference drugs. Standard antibiotics (ciprofloxacin, gentamicin; data not shown) and internal quality control/reference strains are routinely included in parallel testing-basic screening of antibacterial activity.

\subsubsection{Antimycobacterial Activity}

The in vitro antimycobacterial activity against Mycobacterium tuberculosis $\mathrm{H}_{37} \mathrm{Rv}(331 / 88 ; M t b$.) and three strains of nontuberculous mycobacteria (Mycobacterium avium 330/88 [resistant to isoniazid, rifamycines, ofloxacin, and ethambutol], Mycobacterium kansasii 235/80 from the Czech National Collection of Type Cultures, and a clinical isolate of M. kansasii 6509/96) was evaluated using a previously reported method [34]. The micromethod for the determination of MIC was used involving the Šula's semisynthetic medium (SEVAC, Prague, Czech Republic). The investigated compounds were added to the medium as solutions in DMSO; the final volume contained 1.0\% DMSO $(v / v)$. The following concentrations were used: 1000, 500, 250, 125, 62.5, 32, 16, 8, 4, 2 and $1 \mu \mathrm{M}$. The MIC were determined after incubation at $37^{\circ} \mathrm{C}$ for 14 and 21 days, for $M$. kansasii strains additionally for 7 days. MIC (in $\mu \mathrm{M}$ ) was the lowest concentration at which the complete inhibition of mycobacterial growth occurred. First-line oral antitubercular drug isoniazid (INH) and parent PABA were involved as reference compounds.

\subsubsection{Antifungal Activity}

The antifungal properties were evaluated against five yeast strains, namely: Candida albicans ATCC 44859, Candida tropicalis 156, Candida krusei E28, Candida glabrata 20/I, Trichosporon asahii 1188 and three strains of filamentous fungi: Aspergillus fumigatus 231, Lichtheimia corymbifera 272, and Trichophyton interdigitale 445. A microdilution broth method was performed according to EUCAST II, III instructions with slight modifications [35,36]. Briefly, tested compounds were dissolved in DMSO and diluted in a twofold manner with RPMI-1640 medium with L-glutamine, supplemented with $2 \%$ glucose $(w / v)$ and buffered to $\mathrm{pH} 7.0$ with 3 morpholinopropane-1-sulfonic acid (all components were purchased from Sigma-Aldrich, USA). The final concentration of DMSO in the tested medium did not exceed $1 \%(v / v)$ of the total solution composition, and it was confirmed that this concentration did not inhibit the fungal growth. Static incubation was performed in the dark and humidified atmosphere, at $35 \pm 2{ }^{\circ} \mathrm{C}$ for 24 and $48 \mathrm{~h}$ (72 and $120 \mathrm{~h}$ for Trichophyton interdigitale). Positive controls consisted of test microbe solely, while negative controls consisted of medium and DMSO. Internal quality control was included too. Visual inspection was used for MIC endpoints evaluation. The experiments were conducted in duplicates. For the results to be valid, the difference in MIC determined from two parallel measurements must not be greater than one step on the dilution scale.

PABA and triazole antimycotic drug fluconazole were involved as the reference compounds. MIC values of fluconazole mean $\mathrm{MIC}_{50}$ values. 


\subsubsection{Cytotoxicity}

HepG2, human hepatocellular carcinoma cells (ATCC HB-8065; passage 5-8) purchased from Health Protection Agency Culture Collections (ECACC, Salisbury, UK) were cultured in Minimum Essentials Eagle Medium supplemented with 10\% fetal bovine serum, 1\% L-glutamine solution and non-essential amino acid solution (Sigma-Aldrich, St. Louis, MO, USA) in a humidified atmosphere containing $5 \% \mathrm{CO}_{2}$ at $37^{\circ} \mathrm{C}$. For subculturing, the cells were harvested after trypsin/EDTA (Sigma-Aldrich, St. Louis, USA) treatment at $37^{\circ} \mathrm{C}$. To evaluate cytotoxicity, the cells treated with the tested substances were used as experimental groups whereas untreated HepG2 cells served as control groups.

The cells were seeded in a density of 10000 cells per well in a 96-well plate. The following day the cells were treated with each of the tested substances dissolved in DMSO (maximal incubation concentration of DMSO was $1 \% v / v$ ). The tested substances were prepared at different incubation concentrations in triplicates according to their solubility. Simultaneously, the controls representing $100 \%$ cell viability, $0 \%$ cell viability (the cells treated with $10 \%$ DMSO), no cell control and vehiculum controls were also involved in triplicates. After $24 \mathrm{~h}$ incubation in a humidified atmosphere containing $5 \% \mathrm{CO}_{2}$ at $37^{\circ} \mathrm{C}$, the reagent from the kit CellTiter 96 AQueous One Solution Cell Proliferation Assay (CellTiter 96; Promega, Fitchburg, WI, USA) was added. After $2 \mathrm{~h}$ incubation at $37^{\circ} \mathrm{C}$ absorbance of samples was recorded at $490 \mathrm{~nm}$ (TECAN, Infinita M200, Grödig, Austria). A standard toxicological parameter $\mathrm{IC}_{50}$ was calculated by nonlinear regression from a semilogarithmic plot of incubation concentration versus percentage of absorbance relative to untreated controls using GraphPad Prism software (version 6; GraphPad Software, Inc., La Jolla, CA, USA). Results of the experiments are presented as inhibitory concentration which reduces the viability of the cell population to $50 \%$ from the maximal viability $\left(\mathrm{IC}_{50}\right)$.

Parent PABA and a cytotoxic/anticancer drugs tamoxifen (as tamoxifen citrate) and cisplatin were involved as the reference drugs.

\section{Results and Discussion}

\subsection{Chemistry}

We synthesized twenty PABA-derived Schiff bases 1a-1t and one "reduced" compound 1u (i.e., 1a with reduced imine double bond). The synthesis of Schiff bases (Scheme 1) involved a condensation of the amino acid with aldehydes in $\mathrm{MeOH}$ under reflux $(3 \mathrm{~h}$ ) or DEE at room temperature (72 h). Exceptionally, an acidic organic catalyst (0.1 equivalent of toluene-4-sulfonic acid monohydrate) was used to promote the reaction (imines $\mathbf{1 f}$ and $\mathbf{1 t}$ ). The yields were from good to excellent (80-96\%). Seventeen Schiff bases were prepared from substituted salicylaldehydes (1a-1q), one (1r) from 5-nitrofuran-2-carbaldehyde (5-nitrofurfural) and three compounds are analogues of 1a: $\mathrm{O}$-methyl derivative $\mathbf{1 s}$, 4-hydroxy isomer $\mathbf{1 t}$ and molecule $1 \mathbf{u}$ without double bond. Aforementioned 4-[(2-hydroxybenzyl)amino]benzoic acid $\mathbf{1} \mathbf{u}$ was obtained by reductive amination from PABA and salicylaldehyde using sodium cyanoborohydride and glacial acetic acid in $\mathrm{MeOH}$ (yield 74\%; Scheme 2). The structures of the aimed compounds are summarized in Table 1. 
Table 1. Antibacterial activity of PABA derivatives 1.

\begin{tabular}{|c|c|c|c|c|c|c|c|c|c|c|c|c|c|c|c|c|c|c|}
\hline \multirow{3}{*}{ Code } & \multirow{3}{*}{$\mathbf{R}^{1}$} & \multirow{3}{*}{$\mathbf{R}^{2}$} & \multicolumn{16}{|c|}{$\operatorname{MIC}[\mu \mathrm{M}]$} \\
\hline & & & \multicolumn{2}{|c|}{ SA } & \multicolumn{2}{|c|}{ MRSA } & \multicolumn{2}{|c|}{ SE } & \multicolumn{2}{|c|}{ EF } & \multicolumn{2}{|c|}{ EC } & \multicolumn{2}{|c|}{ KP } & \multicolumn{2}{|c|}{ KP-E } & \multicolumn{2}{|c|}{ PA } \\
\hline & & & $24 \mathrm{~h}$ & $48 \mathrm{~h}$ & $24 \mathrm{~h}$ & $48 \mathrm{~h}$ & $24 \mathrm{~h}$ & $48 \mathrm{~h}$ & $24 \mathrm{~h}$ & $48 \mathrm{~h}$ & $24 \mathrm{~h}$ & $48 \mathrm{~h}$ & $24 \mathrm{~h}$ & $48 \mathrm{~h}$ & $24 \mathrm{~h}$ & $48 \mathrm{~h}$ & $24 \mathrm{~h}$ & $48 \mathrm{~h}$ \\
\hline $1 \mathrm{a}$ & $\mathrm{H}$ & $\mathrm{H}$ & $>500$ & $>500$ & $>500$ & $>500$ & $>500$ & $>500$ & $>500$ & $>500$ & $>500$ & $>500$ & $>500$ & $>500$ & $>500$ & $>500$ & $>500$ & $>500$ \\
\hline $1 b$ & $5-\mathrm{F}$ & $\mathrm{H}$ & $>500$ & $>500$ & $>500$ & $>500$ & $>500$ & $>500$ & $>500$ & $>500$ & $>500$ & $>500$ & $>500$ & $>500$ & $>500$ & $>500$ & $>500$ & $>500$ \\
\hline 1c & $5-\mathrm{Cl}$ & $\mathrm{H}$ & $>500$ & $>500$ & $>500$ & $>500$ & 62.5 & 62.5 & $>500$ & $>500$ & $>500$ & $>500$ & $>500$ & $>500$ & $>500$ & $>500$ & $>500$ & $>500$ \\
\hline 1d & $5-\mathrm{Br}$ & $\mathrm{H}$ & $>250$ & $>250$ & $>250$ & $>250$ & 62.5 & 62.5 & $>250$ & $>250$ & $>250$ & $>250$ & $>250$ & $>250$ & $>250$ & $>250$ & $>250$ & $>250$ \\
\hline $1 e$ & $5-\mathrm{I}$ & $\mathrm{H}$ & $>250$ & $>250$ & 250 & 250 & 62.5 & 62.5 & $>250$ & $>250$ & $>250$ & $>250$ & $>250$ & $>250$ & $>250$ & $>250$ & $>250$ & $>250$ \\
\hline 1f & $5-\mathrm{NO}_{2}$ & $\mathrm{H}$ & 500 & 500 & 500 & 500 & 250 & 250 & 500 & 500 & $>500$ & $>500$ & $>500$ & $>500$ & $>500$ & $>500$ & $>500$ & $>500$ \\
\hline $1 \mathrm{~g}$ & $5-\mathrm{CH}_{3}$ & $\mathrm{H}$ & $>500$ & $>500$ & $>500$ & $>500$ & 250 & 250 & $>500$ & $>500$ & $>500$ & $>500$ & $>500$ & $>500$ & $>500$ & $>500$ & $>500$ & $>500$ \\
\hline 1h & $5-\mathrm{OCH}_{3}$ & $\mathrm{H}$ & $>500$ & $>500$ & $>500$ & $>500$ & 500 & 500 & $>500$ & $>500$ & $>500$ & $>500$ & $>500$ & $>500$ & $>500$ & $>500$ & $>500$ & $>500$ \\
\hline $1 \mathrm{i}$ & $5-\mathrm{OH}$ & $\mathrm{H}$ & 500 & 500 & 250 & 250 & 250 & 250 & 500 & 500 & $>500$ & $>500$ & 500 & 500 & 500 & 500 & 500 & 500 \\
\hline $1 j$ & 5-tert-Bu & $\mathrm{H}$ & $>500$ & $>500$ & $>500$ & $>500$ & $>500$ & $>500$ & $>500$ & $>500$ & $>500$ & $>500$ & $>500$ & $>500$ & $>500$ & $>500$ & $>500$ & $>500$ \\
\hline $1 \mathrm{k}$ & 6-Cl & $\mathrm{H}$ & $>500$ & $>500$ & $>500$ & $>500$ & $>500$ & $>500$ & $>500$ & $>500$ & $>500$ & $>500$ & $>500$ & $>500$ & $>500$ & $>500$ & $>500$ & $>500$ \\
\hline 11 & $4-\mathrm{Cl}$ & $\mathrm{H}$ & $>500$ & $>500$ & $>500$ & $>500$ & $>500$ & $>500$ & $>500$ & $>500$ & $>500$ & $>500$ & $>500$ & $>500$ & $>500$ & $>500$ & $>500$ & $>500$ \\
\hline $1 \mathrm{~m}$ & $\mathrm{H}$ & $\mathrm{Cl}$ & $>500$ & $>500$ & $>500$ & $>500$ & 500 & 500 & $>500$ & $>500$ & $>500$ & $>500$ & $>500$ & $>500$ & $>500$ & $>500$ & $>500$ & $>500$ \\
\hline 1n & $5-\mathrm{Cl}$ & $\mathrm{Cl}$ & 125 & 125 & 250 & 250 & 250 & 250 & $>500$ & $>500$ & $>500$ & $>500$ & $>500$ & $>500$ & $>500$ & $>500$ & $>500$ & $>500$ \\
\hline 10 & $5-\mathrm{Cl}$ & $\mathrm{Br}$ & 15.62 & 15.62 & 31.25 & 31.25 & 15.62 & 31.25 & 62.5 & 62.5 & 500 & 500 & 500 & 500 & 500 & 500 & $>500$ & $>500$ \\
\hline $1 p$ & $5-\mathrm{Cl}$ & I & 15.62 & 15.62 & 15.62 & 15.62 & 31.25 & 31.25 & 62.5 & 62.5 & 62.5 & 62.5 & 125 & 125 & 125 & 125 & $>500$ & $>500$ \\
\hline $1 \mathrm{q}$ & 5-I & I & 62.5 & 62.5 & 62.5 & 62.5 & 62.5 & 62.5 & 500 & 500 & 500 & 500 & 500 & 500 & 500 & 500 & $>500$ & $>500$ \\
\hline $1 \mathrm{r}$ & - & - & 62.5 & 62.5 & 62.5 & 62.5 & 125 & 125 & $>500$ & $>500$ & $>500$ & $>500$ & 500 & 500 & 500 & 500 & 500 & 500 \\
\hline 1s & - & - & $>500$ & $>500$ & $>500$ & $>500$ & $>500$ & $>500$ & $>500$ & $>500$ & $>500$ & $>500$ & $>500$ & $>500$ & $>500$ & $>500$ & $>500$ & $>500$ \\
\hline $1 \mathrm{t}$ & - & - & $>500$ & $>500$ & $>500$ & $>500$ & $>500$ & $>500$ & $>500$ & $>500$ & $>500$ & $>500$ & $>500$ & $>500$ & $>500$ & $>500$ & $>500$ & $>500$ \\
\hline $1 \mathrm{u}$ & - & - & $>500$ & $>500$ & $>500$ & $>500$ & $>500$ & $>500$ & $>500$ & $>500$ & $>500$ & $>500$ & $>500$ & $>500$ & $>500$ & $>500$ & $>500$ & $>500$ \\
\hline $\begin{array}{c}\text { PABA } \\
1\end{array}$ & - & - & $>500$ & $>500$ & $>500$ & $>500$ & $>500$ & $>500$ & $>500$ & $>500$ & $>500$ & $>500$ & $>500$ & $>500$ & $>500$ & $>500$ & $>500$ & $>500$ \\
\hline BAC & - & - & 7.81 & 15.62 & 15.62 & 15.62 & 15.62 & 31.25 & 15.62 & 62.5 & $>500$ & $>500$ & $>500$ & $>500$ & $>500$ & $>500$ & $>500$ & $>500$ \\
\hline NEO & - & - & ND & ND & ND & ND & ND & ND & ND & ND & 3.9 & 3.9 & 1.95 & 1.95 & 3.9 & 3.9 & 7.81 & 15.62 \\
\hline
\end{tabular}

BAC = bacitracin; NEO = neomycin; PABA = 4-aminobenzoic acid. SA: Staphylococcus aureus CCM 4516/08; MRSA: methicillin-resistant Staphylococcus aureus H 5996/08; SE: Staphylococcus epidermidis H 6966/08; EF: Enterococcus faecalis J 14365/08. EC: Escherichia coli CCM 4517; KP: Klebsiella pneumoniae D 11750/08; KP-E: ESBL-positive Klebsiella pneumoniae J 14368/08; PA: Pseudomonas aeruginosa CCM 1961. ND = not determined. One or two of the best MIC value(s) for each strain are shown in bold. 


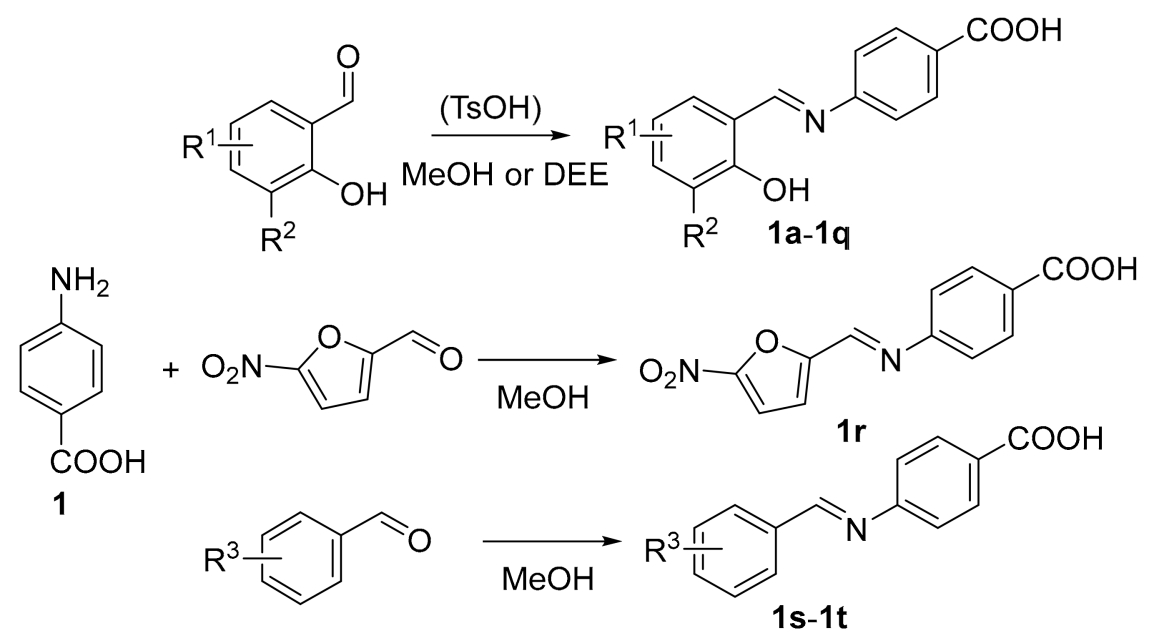

Scheme 1. Synthesis of PABA-based imines 1a-1t $\left(\mathrm{R}^{1}=\mathrm{H}, 5-\mathrm{F}, 4-\mathrm{Cl}, 5-\mathrm{Cl}, 6-\mathrm{Cl}, 5-\mathrm{Br}, 5-\mathrm{I}, 5-\mathrm{NO}_{2}, 5-\mathrm{CH}_{3}\right.$, $5-\mathrm{OCH}_{3}, 5-\mathrm{OH}, 5-t-\mathrm{Bu} ; \mathrm{R}^{2}=\mathrm{H}, \mathrm{Cl}, \mathrm{Br}, \mathrm{I} ; \mathrm{R}^{3}=2-\mathrm{OCH}_{3}, 4-\mathrm{OH}$; TsOH = toluene-4-sulfonic acid; $\mathrm{DEE}=$ diethyl ether).<smiles>Nc1ccc(C(=O)O)cc1</smiles>

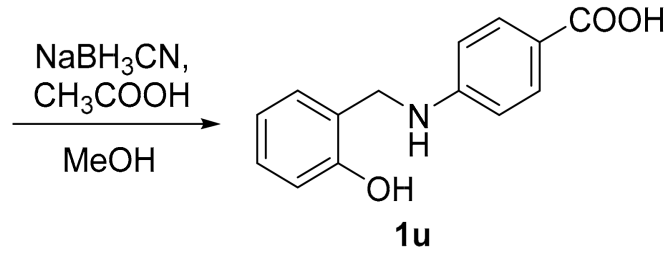

Scheme 2. Synthesis of the derivative $1 \mathbf{u}$ via reductive amination.

New compounds were characterized by melting points, NMR and IR spectroscopy. Their purity was checked by the TLC and elemental analysis additionally. The identity of the known compounds was established using NMR and IR spectroscopy by the comparison with previously reported data. Their purity was checked in an identical way as for novel derivatives.

\subsection{Antibacterial Activity}

PABA derivatives 1a-1u were evaluated for their antibacterial action using four Gram-positive strains of Staphylococcus aureus ATCC 29213, CCM 4223, methicillin-resistant Staphylococcus aureus ATCC 43300, CCM 4750 (MRSA), Staphylococcus epidermidis H 6966/08, Enterococcus faecalis ATCC 29212, CCM 4224 and four Gram-negative strains, Escherichia coli ATCC 25922, CCM 3954, Klebsiella pneumoniae D 11750/08, extended spectrum $\beta$-lactamase positive K. pneumoniae J 14368/08, and Pseudomonas aeruginosa ATCC 27853, CCM 3955 (Table 1). Bacitracin, neomycin and PABA itself were used for comparison of MIC, ciprofloxacin and gentamicin as internal quality standards (their MIC data are not shown).

Free PABA lacked any antibacterial properties (MIC > $500 \mu \mathrm{M})$. Generally, Gram-positive strains were more susceptible (MIC from $15.62 \mu \mathrm{M})$ than Gram-negative species $(\geq 62.5 \mu \mathrm{M}$ and majority of the compounds 1 share no activity). Illustratively, P. aeruginosa was inhibited only by two imines at $500 \mu \mathrm{M}$ (1i and 1r). On the other hand, S. epidermidis was found being the most susceptible strain $(\geq 15.62 \mu \mathrm{M})$ followed by both drug-susceptible and methicillin-resistant $S$. aureus. Importantly, the growth inhibition of $S$. aureus is independent of the presence of methicillin-resistance, thus indicating no cross-resistance with this drug. The MIC values obtained for the most active imines are identical to those obtained for bacitracin, an antibiotic used for the treatment of topical infections caused by Gram-positive bacteria. Due to a lack of a significant activity of $\mathbf{1 a - 1 u}$ against Gram-negative strains, none of the derivatives were superior to aminoglycoside drug neomycin.

The following structure-activity relationships (SAR) were identified: 
Substituted salicylidene moiety is essential: derivatives of unsubstituted salicylaldehyde (1a) and its reduced analogue 1u, 2-methoxybenzaldehyde (1s) and 4-hydroxybenzaldehyde (1t) avoided any antibacterial action.

1. The presence of 5-F (1) $), 5-t-B u(\mathbf{1 j})$ and chlorine at the positions 4 and 6 of the salicylic ring (1k, 11) did not improve activity of $\mathbf{1 a}$.

2. Nitro (1f) and hydroxy group (1i) with opposite electron properties led to similar results-inhibition of multiple Gram-positive strains including MRSA, but at high concentrations $(\geq 250 \mu \mathrm{M})$.

3. The 5-substitution by a single halogen ( $\mathrm{Cl}$ 1c, $\mathrm{Br}$ 1d, I 1e) improves the inhibition of S. epidermidis; in the case of 1e MRSA, too (MIC from $62.5 \mu \mathrm{M}$ ).

4. Electron-donation groups $\left(\mathrm{CH}_{3} \mathbf{1 g}, \mathrm{OCH}_{3} \mathbf{1 h}\right)$ together with 3-Cl (1m) produced inhibition of only S. epidermidis, but with MIC from $250 \mu \mathrm{M}$.

5. 5-Nitrofurylidene PABA derivative 1r inhibited all three staphylococcal strains (MIC 62.5-125 $\mu \mathrm{M})$.

6. The Schiff bases derived from 3,5-dihalogenosalicylaldehydes (1n-1q) represent the most successful modification of PABA with MIC values of $\geq 15.62 \mu \mathrm{M}$, especially if the particular halogens are different $(\mathbf{1 0}, \mathbf{1 p})$. These latter compounds containing one iodine atom also demonstrated activity against three Gram-negative strains (E. coli, K. pneumoniae).

In summary, the presence of at least one heavier halogen is essential, favouring derivatives of 5-chloro-3-iodo/bromo-salicylaldehydes. These substituents provide both wide-spectrum antibacterial activity and comparatively low MIC values. Interestingly, similar results were obtained for sulfadiazine Schiff bases previously [20]. MIC of 5-nitrofurfural-derived Schiff base 1r (62.5-125 $\mu$ M, i.e., 16.25-32.51 $\mu \mathrm{g} / \mathrm{mL}$ ) is fully comparable to breakpoint of its clinically used analogue nitrofurantoin for staphylococci (32-64 $\mu \mathrm{g} / \mathrm{mL}$ ) according to EUCAST [33].

Although PABA is an essential molecule for folate biosynthesis, it was reported as an agent inhibiting E. coli, Listeria monocytogenes and Salmonella enteritidis [6], but at supraphysiological concentrations. Although the strains used by Richards et al. are distinct from our study, their reported MIC values of PABA were substantially higher (9-24 mM) [6]. Giving together with the ineffectiveness of PABA in our assay, it is obvious that the antibacterial action depends on the presence of imine bond resulting from the reaction with substituted salicylaldehydes or 5-nitrofurfural, i.e., the bioactivity is established by this simple chemical modification of the vitamin unequivocally. The substitution of $\mathrm{NH}_{2}$ group by carbonyl group could interfere with the vitamin function of PABA and thus folate biosynthesis. Alternatively, PABA may be considered as a carrier for intrinsic bioactive salicylic and nitrofuran scaffolds. From this point of view, PABA that has demonstrated selective bacterial uptake (S. aureus, E. coli, M. tuberculosis) [37] should help to accumulate Schiff bases and thus salicylic and nitrofuran molecules within bacterial cells where they can disrupt cellular functions leading to growth inhibition or cellular death.

\subsection{Antimycobacterial Activity}

Schiff bases $1 \mathbf{a}-\mathbf{1 t}$ and phenol $\mathbf{1 u}$ were screened in vitro against $M t b$. 331/88 (i.e., $\mathrm{H}_{37} \mathrm{Rv}$ ) and three non-tuberculous mycobacterial strains: M. avium 330/88, M. kansasii 235/80 and M. kansasii $6509 / 96$ (a clinical isolate). A first-line antitubercular drug INH and parent PABA were used as reference drugs for comparison (Table 2). 
Table 2. Antimycobacterial activity of PABA derivatives 1.

\begin{tabular}{|c|c|c|c|c|c|c|c|c|c|c|c|c|}
\hline \multirow{3}{*}{ Code } & \multirow{3}{*}{$\mathbf{R}$} & \multirow{3}{*}{$\mathbf{R}^{2}$} & \multicolumn{10}{|c|}{$\operatorname{MIC}[\mu \mathrm{M}]$} \\
\hline & & & \multicolumn{2}{|c|}{ Mtb. 331/88 } & \multicolumn{2}{|c|}{ M. avium 330/88 } & \multicolumn{3}{|c|}{ M. kansasii 235/80 } & \multicolumn{3}{|c|}{ M. kansasii 6509/96 } \\
\hline & & & $14 \mathrm{~d}$ & $21 \mathrm{~d}$ & $14 \mathrm{~d}$ & $21 \mathrm{~d}$ & $7 \mathrm{~d}$ & $14 \mathrm{~d}$ & $21 \mathrm{~d}$ & $7 \mathrm{~d}$ & $14 \mathrm{~d}$ & $21 \mathrm{~d}$ \\
\hline 1a & $\mathrm{H}$ & $\mathrm{H}$ & $>1000$ & $>1000$ & $>1000$ & $>1000$ & 1000 & $>1000$ & $>1000$ & 1000 & 1000 & 1000 \\
\hline $1 \mathrm{~b}$ & $5-\mathrm{F}$ & $\mathrm{H}$ & 500 & 500 & $>1000$ & $>1000$ & 250 & 500 & 500 & 500 & 500 & 500 \\
\hline 1c & $5-\mathrm{Cl}$ & $\mathrm{H}$ & 250 & 500 & 1000 & $>1000$ & 250 & 250 & 500 & 500 & 500 & 1000 \\
\hline 1d & $5-\mathrm{Br}$ & $\mathrm{H}$ & 250 & 500 & 500 & 500 & 250 & 250 & 500 & 500 & 500 & 500 \\
\hline $1 \mathrm{e}$ & $5-\mathrm{I}$ & $\mathrm{H}$ & 250 & 250 & 500 & 500 & 250 & 250 & 250 & 250 & 250 & 500 \\
\hline 1f & $5-\mathrm{NO}_{2}$ & $\mathrm{H}$ & 250 & 500 & 1000 & $>1000$ & 500 & 500 & 500 & 500 & 500 & 500 \\
\hline $1 \mathrm{~g}$ & $5-\mathrm{CH}_{3}$ & $\mathrm{H}$ & 500 & 500 & $>1000$ & $>1000$ & 500 & 500 & 500 & 500 & 500 & 1000 \\
\hline $1 \mathrm{~h}$ & $5-\mathrm{OCH}_{3}$ & $\mathrm{H}$ & 250 & 500 & 1000 & 1000 & 500 & 500 & 500 & 500 & 500 & 500 \\
\hline $1 \mathrm{i}$ & $5-\mathrm{OH}$ & $\mathrm{H}$ & 500 & 1000 & 1000 & $>1000$ & 500 & 500 & 1000 & 500 & 1000 & 1000 \\
\hline $\mathbf{1 j}$ & 5-tert-Bu & $\mathrm{H}$ & 250 & 250 & 1000 & $>1000$ & 125 & 125 & 250 & 250 & 250 & 500 \\
\hline $1 \mathrm{k}$ & 6-Cl & $\mathrm{H}$ & 250 & 500 & 500 & 1000 & 250 & 500 & 500 & 250 & 250 & 500 \\
\hline 11 & $4-\mathrm{Cl}$ & $\mathrm{H}$ & 250 & 500 & 1000 & 1000 & 250 & 500 & 500 & 250 & 250 & 500 \\
\hline $1 \mathrm{~m}$ & $\mathrm{H}$ & $\mathrm{Cl}$ & 500 & 500 & 1000 & 1000 & 500 & 500 & 500 & 250 & 500 & 500 \\
\hline $1 n$ & $5-\mathrm{Cl}$ & $\mathrm{Cl}$ & 500 & 500 & 1000 & 1000 & 500 & 500 & 500 & 250 & 500 & 500 \\
\hline 10 & $5-\mathrm{Cl}$ & $\mathrm{Br}$ & 250 & 500 & 500 & 500 & 250 & 500 & 500 & 500 & 500 & 500 \\
\hline $1 p$ & $5-\mathrm{Cl}$ & I & 250 & 250 & 500 & 1000 & 250 & 500 & 500 & 250 & 250 & 500 \\
\hline $1 q$ & $5-\mathrm{I}$ & I & 250 & 250 & 500 & 500 & 250 & 500 & 500 & 250 & 250 & 250 \\
\hline $1 \mathrm{r}$ & - & - & 62.5 & 125 & 250 & 500 & 62.5 & 125 & 250 & 125 & 125 & 250 \\
\hline $1 \mathrm{~s}$ & - & - & 1000 & 1000 & $>1000$ & $>1000$ & 1000 & 1000 & $>1000$ & 1000 & 1000 & 1000 \\
\hline $1 t$ & - & - & 1000 & 1000 & 1000 & $>1000$ & 1000 & 1000 & $>1000$ & 1000 & 1000 & 1000 \\
\hline $1 u$ & - & - & 1000 & 1000 & $>1000$ & $>1000$ & 1000 & 1000 & 1000 & 1000 & 1000 & 1000 \\
\hline PABA 1 & - & - & $>1000$ & $>1000$ & $>1000$ & $>1000$ & 1000 & $>1000$ & $>1000$ & 1000 & 1000 & 1000 \\
\hline INH & - & - & 0.5 & 1 & $>250$ & $>250$ & $>250$ & $>250$ & $>250$ & 8 & 8 & 8 \\
\hline
\end{tabular}

$\mathrm{INH}=$ isoniazid; PABA = 4-aminobenzoic acid; Mtb. = Mycobacterium tuberculosis. One or two of the lowest MIC value(s) for each strain are shown in bold. 
In general, although none of the compounds was inactive completely, antimycobacterial activity of the derivatives 1a-1u is comparatively weak with MIC from $62.5 \mu \mathrm{M}$. 5-nitrofurfural derivative $\mathbf{1 r}$ showed the lowest MIC (62.5-250 $\mu \mathrm{M})$; only this derivative outstripped INH in the case of M. avium and M. kansasii 235/80 (i.e., INH-resistant strains). This finding was expected regarding previous studies, e.g., [38]. The Schiff bases derived from unsubstituted salicylaldehyde (1a), its 4-OH isomer (1t), 2-methoxybenzaldehyde (1s) and reduced compound $\mathbf{1} \mathbf{u}$ share only negligible antimycobacterial activity corresponding to the parent PABA. The substitution of salicylaldehyde (compounds $\mathbf{1} \mathbf{b}-\mathbf{1 q}$; especially tert-butyl $\mathbf{1} \mathbf{j}$ and halogenated imines $\mathbf{1} \mathbf{i}, \mathbf{1 k}, \mathbf{1} \mathbf{1}, \mathbf{1} \mathbf{p}$ and $\mathbf{1 q}$ ) improved growth inhibition of mycobacteria, but only moderately.

\subsection{Antifungal Activity}

PABA derivatives 1a-1u were investigated also against eight fungal strains. The panel of human pathogens covers five yeast strains (Candida albicans ATCC 44859, Candida tropicalis 156, Candida krusei E28, Candida glabrata 20/I, Trichosporon asahii 1188) and three moulds (Aspergillus fumigatus 231, Lichtheimia corymbifera 272, and Trichophyton interdigitale 445). Azole drug fluconazole and parent PABA were used for comparison of MIC (Table 3).

PABA itself showed no antifungal properties (MIC $>500 \mu \mathrm{M}$ ). In general, A. fumigatus and $L$. corymbifera were found being the most resistant species (MIC from $125 \mu \mathrm{M}$ ). On the other hand, yeasts and T. interdigitale were inhibited from the concentration of $7.81 \mu \mathrm{M}$. Interestingly, the inhibition of these strains by particular derivatives were virtually identical. The PABA Schiff bases were superior to fluconazole for seven strains: C. tropicalis $(\mathbf{1} \mathbf{c}-\mathbf{1 e}, \mathbf{1 h}, \mathbf{1 k}, \mathbf{1} \mathbf{m}-\mathbf{1 r})$, C. krusei (inherent FLU-resistant; (1e, 1o-1r; other derivatives were comparable-1c, 1d, 1k, 1m, and $\mathbf{1 n})$, C. glabrata (1d, 1e, 1m, 1o-1q), T. asahii $\mathbf{( 1 d}, \mathbf{1 e}, \mathbf{1 m}-\mathbf{1 q}$; others are comparable: $\mathbf{1 f}, \mathbf{1 h}, \mathbf{1 r})$, A. fumigatus $(\mathbf{1 d}, \mathbf{1 e}, \mathbf{1 m}-\mathbf{1 q})$, L. corymbifera $(\mathbf{1} \mathbf{f}$, 1m-1q) and T. interdigitale (1p, 1q; additional derivatives were superior or comparable after $120 \mathrm{~h}$ of treatment).

These SAR were found:

1. Substituted salicylidene or 5-nitrofurfurylidene moiety is indispensable: unsubstituted salicylaldehyde (1a), its reduced analogue 1u, 2-methoxybenzaldehyde (1s) and 4-hydroxybenzaldehyde (1t) share no antimycotic potency.

2. 5-F (1) $)$ and 5-alkyls $(\mathbf{1 g}, \mathbf{1 j})$ are not translated into any antifungal action.

3. The presence of $\mathrm{NO}_{2}(\mathbf{1 f}), \mathrm{OH}(\mathbf{1 i}), \mathrm{OCH}_{3}(\mathbf{1 h})$ and $4-\mathrm{Cl}(\mathbf{1 1})$ substituents provides active compounds but with higher MIC values $(\geq 250 \mu \mathrm{M})$.

4. Focusing on isomeric 6- $\mathrm{Cl}(\mathbf{1 k}), 5-\mathrm{Cl}(\mathbf{1 c}), 4-\mathrm{Cl}(\mathbf{1 1})$ and 3- $\mathrm{Cl}(\mathbf{1 m})$ derivatives, the increase of the activity is as follows: $4-\mathrm{Cl}$ ( $\mathrm{MIC} \geq 250 \mu \mathrm{M}$ ) $<5-\mathrm{Cl} \leq 6-\mathrm{Cl}<3-\mathrm{Cl}$ (MIC $\geq 31.25 \mu \mathrm{M}$ ). Interestingly, the introduction of second chlorine atom $\left(3,5-\mathrm{Cl}_{2}\right)$ did not improve the activity $(\mathbf{1 n} ; \mathrm{MIC} \geq 125$ $\mu \mathrm{M})$.

5. The activity of single halogen-substituted derivatives (5-F $\mathbf{1 b}, 5-\mathrm{Cl} \mathbf{1 c}, 5-\mathrm{Br} \mathbf{1 d}, 5-\mathrm{I} \mathbf{1 e}$ ) is increasing with the atomic mass/lipophilicity/atomic radius of the halogens (5-F > $500 \mu \mathrm{M} ; 5-\mathrm{I} \geq 31.25 \mu \mathrm{M})$.

6. 3,5-Dihalogenosalicylidene imines (1n-1q) were identified as the most active derivatives providing both low MIC $(\geq 7.81 \mu \mathrm{M})$ and broad-spectrum of the activity. The presence of at least one iodine atom results in the highest antifungal properties (1o and $\mathbf{1 p})$.

Obviously, SAR are predominantly analogous to antibacterial action. Recently, Laborda et al. [39] have discovered PABA as antifungal substance of Lysobacter antibioticus supressing phytopathogenic fungi, even though at high concentrations again ( $\mathrm{IC}_{50}$ of 1-3 $\mathrm{mM}$ ), i.e., being up to three orders of magnitude less potent then our compounds. The maximal concentration of PABA investigated in our study was lower than reported as effective [39]. To the best of our knowledge, no activity of PABA-based imines working against human pathogenic fungi has been reported and this property is presented herein for the first time. Again, a simple structural change of this vitamin-like molecule constitutes new bioactivity. 
Table 3. Antifungal activity of PABA derivatives 1 .

\begin{tabular}{|c|c|c|c|c|c|c|c|c|c|c|c|c|c|c|c|c|c|c|}
\hline \multirow{3}{*}{ Code } & \multirow{3}{*}{$\mathbf{R}^{1}$} & \multirow{3}{*}{$\mathbf{R}^{2}$} & \multicolumn{16}{|c|}{$\mathrm{MIC}[\mu \mathrm{M}]$} \\
\hline & & & \multicolumn{2}{|c|}{ CA } & \multicolumn{2}{|c|}{ CT } & \multicolumn{2}{|c|}{ CK } & \multicolumn{2}{|c|}{ CG } & \multicolumn{2}{|c|}{ TA } & \multicolumn{2}{|c|}{ AF } & \multicolumn{2}{|c|}{ LC } & \multicolumn{2}{|c|}{ TI } \\
\hline & & & $24 \mathrm{~h}$ & $48 \mathrm{~h}$ & $24 \mathrm{~h}$ & $48 \mathrm{~h}$ & $24 \mathrm{~h}$ & $48 \mathrm{~h}$ & $24 \mathrm{~h}$ & $48 \mathrm{~h}$ & $24 \mathrm{~h}$ & $48 \mathrm{~h}$ & $24 \mathrm{~h}$ & $48 \mathrm{~h}$ & $24 \mathrm{~h}$ & $48 \mathrm{~h}$ & $72 \mathrm{~h}$ & $120 \mathrm{~h}$ \\
\hline 1a & $\mathrm{H}$ & $\mathrm{H}$ & $>500$ & $>500$ & $>500$ & $>500$ & $>500$ & $>500$ & $>500$ & $>500$ & $>500$ & $>500$ & $>500$ & $>500$ & $>500$ & $>500$ & $>500$ & $>500$ \\
\hline $1 b$ & $5-\mathrm{F}$ & $\mathrm{H}$ & $>500$ & $>500$ & $>500$ & $>500$ & $>500$ & $>500$ & $>500$ & $>500$ & $>500$ & $>500$ & $>500$ & $>500$ & $>500$ & $>500$ & $>500$ & $>500$ \\
\hline 1c & $5-\mathrm{Cl}$ & $\mathrm{H}$ & $>125$ & $>125$ & 125 & 125 & 125 & 125 & 125 & 125 & $>125$ & $>125$ & $>125$ & $>125$ & $>125$ & $>125$ & 125 & 125 \\
\hline 1d & $5-\mathrm{Br}$ & $\mathrm{H}$ & 62.5 & 62.5 & 125 & 125 & 125 & 125 & 62.5 & 62.5 & 125 & 125 & 250 & 250 & $>250$ & $>250$ & 62.5 & 62.5 \\
\hline 1e & $5-\mathrm{I}$ & $\mathrm{H}$ & 62.5 & 62.5 & 125 & 125 & 62.5 & 62.5 & 31.25 & 31.25 & 62.5 & 62.5 & 125 & 125 & $>125$ & $>125$ & 31.25 & 31.25 \\
\hline 1f & $5-\mathrm{NO}_{2}$ & $\mathrm{H}$ & 500 & 500 & $>500$ & $>500$ & $>500$ & $>500$ & $>500$ & $>500$ & 500 & 500 & $>500$ & $>500$ & 500 & 500 & 500 & 500 \\
\hline $1 \mathrm{~g}$ & $5-\mathrm{CH}_{3}$ & $\mathrm{H}$ & $>125$ & $>125$ & $>125$ & $>125$ & $>125$ & $>125$ & $>125$ & $>125$ & $>125$ & $>125$ & $>125$ & $>125$ & $>125$ & $>125$ & $>125$ & $>125$ \\
\hline $1 \mathrm{~h}$ & $5-\mathrm{OCH}_{3}$ & $\mathrm{H}$ & 250 & 250 & 500 & 500 & 500 & 500 & 250 & 250 & 500 & 500 & $>500$ & $>500$ & $>500$ & $>500$ & $>500$ & $>500$ \\
\hline $1 \mathrm{i}$ & $5-\mathrm{OH}$ & $\mathrm{H}$ & 500 & 500 & $>500$ & $>500$ & $>500$ & $>500$ & 250 & 250 & $>500$ & $>500$ & $>500$ & $>500$ & $>500$ & $>500$ & $>500$ & $>500$ \\
\hline $\mathbf{1 j}$ & 5-tert-Bu & $\mathrm{H}$ & $>500$ & $>500$ & $>500$ & $>500$ & $>500$ & $>500$ & $>500$ & $>500$ & $>500$ & $>500$ & $>500$ & $>500$ & $>500$ & $>500$ & $>500$ & $>500$ \\
\hline $1 \mathrm{k}$ & 6-Cl & $\mathrm{H}$ & 125 & 125 & 62.5 & 125 & 125 & 250 & 125 & 125 & 125 & $>125$ & $>125$ & $>125$ & $>125$ & $>125$ & 125 & 125 \\
\hline 11 & $4-\mathrm{Cl}$ & $\mathrm{H}$ & 250 & $>500$ & 500 & $>500$ & 500 & $>500$ & 500 & $>500$ & 500 & $>500$ & 250 & $>500$ & $>500$ & $>500$ & 500 & $>500$ \\
\hline $1 \mathrm{~m}$ & $\mathrm{H}$ & $\mathrm{Cl}$ & 31.25 & 31.25 & 62.5 & 62.5 & 250 & 250 & 62.5 & 62.5 & 125 & 125 & 250 & 250 & 500 & 500 & 125 & 125 \\
\hline 1n & $5-\mathrm{Cl}$ & $\mathrm{Cl}$ & 125 & 125 & 250 & 250 & 125 & 125 & 125 & 125 & 125 & 125 & 500 & 500 & 500 & 500 & 250 & 250 \\
\hline 10 & $5-\mathrm{Cl}$ & $\mathrm{Br}$ & 31.25 & 31.25 & 62.5 & 62.5 & 62.5 & 62.5 & 15.62 & 15.62 & 31.25 & 31.25 & 250 & 250 & 500 & 500 & 62.5 & 62.5 \\
\hline $1 p$ & $5-\mathrm{Cl}$ & I & 7.81 & 7.81 & 31.25 & 31.25 & 15.62 & 15.62 & 7.81 & 7.81 & 7.81 & 7.81 & 125 & 125 & 250 & 250 & 7.81 & 7.81 \\
\hline $1 \mathrm{q}$ & 5-I & I & 7.81 & 7.81 & 15.62 & 15.62 & 31.25 & 31.25 & 7.81 & 7.81 & 15.62 & 15.62 & 125 & 125 & 250 & 250 & 7.81 & 7.81 \\
\hline $1 \mathrm{r}$ & - & - & 250 & 250 & 250 & 250 & 62.5 & 62.5 & 250 & 250 & 500 & 500 & $>500$ & $>500$ & $>500$ & $>500$ & 250 & 250 \\
\hline 1s & - & - & $>500$ & $>500$ & $>500$ & $>500$ & $>500$ & $>500$ & $>500$ & $>500$ & $>500$ & $>500$ & $>500$ & $>500$ & $>500$ & $>500$ & $>500$ & $>500$ \\
\hline $1 t$ & - & - & $>500$ & $>500$ & $>500$ & $>500$ & $>500$ & $>500$ & $>500$ & $>500$ & $>500$ & $>500$ & $>500$ & $>500$ & $>500$ & $>500$ & $>500$ & $>500$ \\
\hline $1 \mathrm{u}$ & - & - & $>500$ & $>500$ & $>500$ & $>500$ & $>500$ & $>500$ & $>500$ & $>500$ & $>500$ & $>500$ & $>500$ & $>500$ & $>500$ & $>500$ & $>500$ & $>500$ \\
\hline $\begin{array}{c}\text { PABA } \\
1\end{array}$ & - & - & $>500$ & $>500$ & $>500$ & $>500$ & $>500$ & $>500$ & $>500$ & $>500$ & $>500$ & $>500$ & $>500$ & $>500$ & $>500$ & $>500$ & $>500$ & $>500$ \\
\hline FLU & - & - & 0.24 & 0.24 & $>500$ & $>500$ & 125 & 250 & 31.25 & 500 & 250 & 500 & $>500$ & $>500$ & $>500$ & $>500$ & 7.81 & 125 \\
\hline
\end{tabular}

FLU = fluconazole; PABA = 4-aminobenzoic acid. CA: Candida albicans ATCC 44859; CT: Candida tropicalis; CK: Candida krusei E28; CG: Candida glabrata 20/I; TA: Trichosporon
1188; AF: Aspergillus fumigatus 231; LC: Lichtheimia corymbifera 272; TI: Trichophyton interdigitale 445. One or two of the best MIC value(s) for each strain are shown in bold. 
Notably, Meir and Osherov [40] presented and highlighted vitamin biosynthesis as promising antifungal targets including folic acid biosynthesis utilizing PABA. The deficient synthesis of PABA and withdrawal from food hampered the virulence of Aspergillus spp. completely. Moreover, PABA inhibitors will likely be active against ongoing infections.

\subsection{Cytotoxicity}

The cytotoxicity of the PABA derivatives 1 were screened using the standard hepatic cancer cell line HepG2 (Table 4) from two reasons. First, salicylidene-based compounds have been proposed as anticancer agents supressing also HepG2 cells [41]. On the other hand, they can serve as an in vitro model for the hepatotoxicity as the surrogate of normal liver cells during preclinical drug discovery $[42,43]$.

Table 4. Cytotoxicity of the compounds 1 for HepG2 cells.

\begin{tabular}{ccccc}
\hline Code & $\mathbf{R}^{\mathbf{1}}$ & $\mathbf{R}^{\mathbf{2}}$ & $\mathbf{I C}_{\mathbf{5 0}}[\boldsymbol{\mu M}]$ & Range of Concentrations Tested \\
\hline $\mathbf{1 a}$ & $\mathrm{H}$ & $\mathrm{H}$ & $>1500 *$ & $1-1500$ \\
$\mathbf{1 b}$ & $5-\mathrm{F}$ & $\mathrm{H}$ & 497.6 & $1-500$ \\
$\mathbf{1 c}$ & $5-\mathrm{Cl}$ & $\mathrm{H}$ & 142.9 & $1-500$ \\
$\mathbf{1 d}$ & $5-\mathrm{Br}$ & $\mathrm{H}$ & 178.7 & $1-500$ \\
$\mathbf{1} \mathbf{d}$ & $5-\mathrm{I}$ & $\mathrm{H}$ & 113.4 & $1-500$ \\
$\mathbf{1 f}$ & $5-\mathrm{NO}_{2}$ & $\mathrm{H}$ & 243.8 & $1-500$ \\
$\mathbf{1 g}$ & $5-\mathrm{CH}_{3}$ & $\mathrm{H}$ & 956.4 & $1-1500$ \\
$\mathbf{1 h}$ & $5-\mathrm{CH}_{3} \mathrm{O}$ & $\mathrm{H}$ & 530.4 & $1-1500$ \\
$\mathbf{1 i}$ & $5-\mathrm{OH}$ & $\mathrm{H}$ & $\mathrm{ND}$ & $1-1500$ \\
$\mathbf{1 j}$ & $5-t e r t-\mathrm{Bu}$ & $\mathrm{H}$ & 80.8 & $1-500$ \\
$\mathbf{1 k}$ & $6-\mathrm{Cl}$ & $\mathrm{H}$ & 279.6 & $1-500$ \\
$\mathbf{1 1}$ & $4-\mathrm{Cl}$ & $\mathrm{H}$ & 263.9 & $1-500$ \\
$\mathbf{1 m}$ & $\mathrm{H}$ & $\mathrm{Cl}$ & 247.4 & $1-500$ \\
$\mathbf{1 n}$ & $5-\mathrm{Cl}$ & $\mathrm{Cl}$ & 98.7 & $1-500$ \\
$\mathbf{1 0}$ & $5-\mathrm{Cl}$ & $\mathrm{Br}$ & 85.7 & $1-500$ \\
$\mathbf{1} \mathbf{1}$ & $5-\mathrm{Cl}$ & $\mathrm{I}$ & 53.4 & $1-500$ \\
$\mathbf{1 q}$ & $5-\mathrm{I}$ & $\mathrm{I}$ & 57.8 & $1-500$ \\
$\mathbf{1 r}$ & - & - & 15.0 & $1-500$ \\
$\mathbf{1 s}$ & - & - & 1090.0 & $1-1500$ \\
$\mathbf{1 t}$ & - & - & $>1500 *$ & $1-1500$ \\
$\mathbf{1 u}$ & - & - & $>500 *$ & $1-500$ \\
Tamoxifen & - & - & 19.6 & $1-500$ \\
Cisplatin & - & - & 21.3 & $1-500$ \\
$\mathbf{P A B A} \mathbf{1}$ & - & - & $>1500 *$ & $1-1500$ \\
\hline
\end{tabular}

* measurement at higher concentration was unable due to the precipitation of the compound in the medium. PABA $=4$-aminobenzoic acid $; \mathrm{ND}=$ not determined.

The CellTiter 96 assay used is based on the reduction of tetrazolium dye in living cells to formazan, which is then determined colorimetrically. This reduction is related to availability of NADH or NADPH. The decline in levels of these metabolically important compounds in the cell causes that the production of formazan is reduced. For quantitative comparison of cytotoxicity, the parameter $\mathrm{IC}_{50}$ was used. $\mathrm{IC}_{50}$ is defined as the concentration that reduces the viability of the cells to $50 \%$ of the maximal viability.

We were able to determine the exact $\mathrm{IC}_{50}$ of the majority of the substances. However, $\mathrm{IC}_{50}$ values of parent PABA 1, 1a, $1 \mathbf{t}$ and $\mathbf{1} \mathbf{u}$ were higher than tested range of concentrations. The measurement was not possible due to the precipitation of the compound in the medium. The $\mathrm{IC}_{50}$ of $\mathbf{1 i}$ could not be determined with the used method due to strong interaction with the testing reagent.

The effective cytotoxic concentrations among the compounds differ in the extent of several orders of magnitude. We could identify both several relatively non-toxic compounds for HepG2 cells $\left(\mathrm{IC}_{50}>500 \mu \mathrm{M}: \mathbf{1 a}, \mathbf{1 g}, \mathbf{1 h}, \mathbf{1 s}, \mathbf{1 t}, \mathbf{1} \mathbf{u}\right.$ and PABA $\left.\mathbf{1}\right)$, other compounds exhibited moderate toxicity. 5-nitrofurylidene-based imine was identified as the most toxic compound $\left(\mathrm{IC}_{50}=15.0 \mu \mathrm{M}\right)$, followed 
by polyhalogenated derivatives and 5-tert-butyl compound $\mathbf{1 j}$. Interestingly, there are not identical SAR as for antimicrobial action: heavier/more lipophilic halogens do not result in uniformly enhanced toxicity and, regarding particular chlorinated isomers, 5-isomer is approximately twice more toxic than other. The $\mathrm{IC}_{50}$ value of the most cytotoxic compound (nitrofuran derivative 1r) was fully comparable to those obtained for anticancer drugs tamoxifen $(19.6 \mu \mathrm{M})$ and cisplatin $(21.3 \mu \mathrm{M})$, other the most active compounds exhibited $\mathrm{IC}_{50}$ in the same order of magnitude $(\mathbf{1} \mathbf{j}, \mathbf{1} \mathbf{n}-\mathbf{1} \mathbf{p})$.

In addition to PABA "non-imine" cytotoxic derivatives [1,5,12,44], Farooq et al. [18] discovered $N$-alkylisatin-3-iminobenzoic acids, i.e., Schiff bases of substituted isatin and PABA, as potential cytotoxic/antileukemic agents with $\mathrm{IC}_{50}$ for human normal as well as cancer cell lines comparable to our the most active molecules. Thus, the formation of Schiff bases could convert non-toxic PABA to moderate cytotoxic agents, depending on the carbonyl compound used. Seemingly negligible changes to the structure may result in substantially higher toxicity for malignant cells (illustratively, switch of a lipophilic and electron-donating substituent methyl $\mathbf{1 g}$ to tert-butyl $\mathbf{1 j}$ increases toxicity almost twelve times). Since PABA as the parent compound itself is non-toxic and safe for human administration [8,9], the cytotoxicity should be a consequence of the properties of the attached aldehyde and/or imine bond.

\section{Conclusions}

In this study, we synthesized twenty-one hybrid compounds of folate precursor 4-aminobenzoic acid, an essential vitamin for many human pathogens, and bioactive aldehydes, predominantly substituted salicylaldehydes and 5-nitrofurfural. These scaffolds were fused through imine bond to form Schiff bases. Advantageously, the aimed compounds are easily synthetically available.

The modification of PABA in this way led to constitution of various bioactivities that were not exhibited by the parent drug in our assays. SAR revealed following essential structural features: (1) presence of imine bond; (2) (5-nitrofuran-2-yl)methylene or 2-hydroxybenzylidene (salicylidene) scaffold; (3) substitution of salicylic moiety, especially by one or more heavier halogen(s). Many of the new compounds share antibacterial action especially against Gram-positive strains including MRSA, mild inhibition of mycobacteria and broad-spectrum antifungal properties. The most potent agents against Gram-positive strains were halogenated, especially 3,5-dihalogenated derivatives with MIC values from $15.62 \mu \mathrm{M}$. The 5-nitrofurfurylidene scaffold was proven to be beneficial for the inhibition of mycobacteria (MIC of $\geq 62.5 \mu \mathrm{M}$ ). Moreover, the simple chemical derivatization of non-toxic parent amino acid can result in compounds with cytotoxic action for cancer cells, but many derivatives remained non-toxic. The presence of heavier halogen(s), especially iodine, highly lipophilic tert-butyl and nitro group ( $\mathrm{IC}_{50}$ from $15.0 \mu \mathrm{M}$ ) enhanced the cytotoxicity of the Schiff bases for HepG2 line. In sum, structure-activity relationships highlighted the presence of dihalogenated salicylic and 5-nitrofurane moieties. Regarding the carbonyl compound used for the modification of the PABA structure, it is possible to tune up the particular activities to identify even more promising derivatives for further development.

Author Contributions: Conceptualization: M.K. and J.V.; Methodology, M.K., K.K., F.T. and J.S.; Investigation, M.K., K.K., J.J., M.B., O.J. and J.S.; Writing-Original Draft Preparation, M.K. and K.K.; Writing-Review and Editing, F.T. and J.V.; Supervision, M.K. and J.V. All authors have read and agreed to the published version of the manuscript.

Funding: This research was funded by the Czech Science Foundation, grant number 17-27514Y, and by the project EFSA-CDN [grant No. CZ.02.1.01/0.0/0.0/16_019/0000841] co-funded by ERDF.

Conflicts of Interest: The authors declare no conflict of interest.

\section{References}

1. Patel, H.M.; Bhardwaj, V.; Sharma, P.; Noolvi, M.N.; Lohan, S.; Bansal, S.; Sharma, A. Quinoxaline-PABA bipartite hybrid derivatization approach: Design and search for antimicrobial agents. J. Mol. Struct. 2019, 1184, 562-568. [CrossRef]

2. Akberova, S.I. New Biological Properties of p-Aminobenzoic Acid. Biol. Bull. 2002, 29, 390-393. [CrossRef] 
3. Kadhum, W.R.; Oshizaka, T.; Ichiro,H.; Todo, H.; Sugibayashi, K. Usefulness of liquid-crystal oral formulations to enhance the bioavailability and skin tissue targeting of $p$-amino benzoic acid as a model compound. Eur. J. Pharm. Sci. 2016, 88, 282-290. [CrossRef] [PubMed]

4. Crisan, M.E.; Bourosh, P.; Maffei, M.E.; Forni, A.; Pieraccini, S.; Sironi, M.; Chumakov, Y.M. Synthesis, Crystal Structure and Biological Activity of 2-Hydroxyethylammonium Salt of p-Aminobenzoic Acid. PLoS One 2014, 9, e101892. [CrossRef] [PubMed]

5. Sowinska, M.; Morawiak, M.; Bochyńska-Czyż, M.; Lipkowski, A.W.; Ziemińska, E.; Zabłocka, B.; Urbanczyk-Lipkowska, Z. Molecular Antioxidant Properties and In Vitro Cell Toxicity of the $p$-Aminobenzoic Acid (PABA) Functionalized Peptide Dendrimers. Biomolecules 2019, 9, 89. [CrossRef] [PubMed]

6. Richards, R.M.; Xing, D.K.; King, T.P. Activity of $p$-aminobenzoic acid compared with other organic acids against selected bacteria. J. Appl. Bacteriol. 1995, 78, 209-215. [CrossRef]

7. Markitantova, Y.V.; Akberova, S.I.; Ryabtseva, A.A.; Stroeva, O.G. Effect of para-Aminobenzoic Acid on Apoptosis Processes in the Adult Rat Conjunctiva and Corneal Epithelium in vivo after Hypobaric Hypoxia. Biol. Bull. Russ. Acad. Sci. 2018, 45, 226-234. [CrossRef]

8. Chang, T.-Y.; Hu, M.-L. Concentrations and lipid peroxidation in tissues and toxicity of para-aminobenzoic acid fed to rats in drinking water. J. Nutr. Biochem. 1996, 7, 408-413. [CrossRef]

9. Nortje, C.; Jansen van Rensburg, P.; Cooke, C.; Erasmus, E. The simultaneous detection and quantification of $p$-aminobenzoic acid and its phase 2 biotransformation metabolites in human urine using LC-MS/MS. Bioanalysis 2015, 7, 1211-1224. [CrossRef]

10. Švarcová, M.; Krátký, M.; Vinšová, J. Investigation of potential inhibitors of chorismate-utilizing enzymes. Curr. Med. Chem. 2015, 22, 1383-1399. [CrossRef]

11. Yun, M.-K.; Wu, Y.; Li, Z.; Zhao, Y.; Waddell, M.B.; Ferreira, A.M.; Lee, R.E.; Bashford, D.; White, S.W. Catalysis and Sulfa Drug Resistance in Dihydropteroate Synthase: Crystal structures reveal the catalytic mechanism of DHPS and the structural basis of sulfa drug action and resistance. Science 2012, 335, 1110-1114. [CrossRef] [PubMed]

12. Kluczyk, A.; Popek, T.; Kiyota, T.; de Macedo, P.; Stefanowicz, P.; Lazar, C.; Konishi, Y. Drug evolution: p-aminobenzoic acid as a building block. Curr. Med. Chem. 2002, 9, 1871-1892. [CrossRef] [PubMed]

13. Beran, J.; Šalapová, E.; Špajdel, M. Inosine pranobex is safe and effective for the treatment of subjects with confirmed acute respiratory viral infections: Analysis and subgroup analysis from a Phase 4, randomised, placebo-controlled, double-blind study. BMC Infect. Dis. 2016, 16, 648. [CrossRef] [PubMed]

14. Zheng, J.; Rubin, E.J.; Bifani, P.; Mathys, V.; Lim, V.; Au, M.; Jang, J.; Nam, J.; Dick, T.; Walker, J.R.; et al. para-Aminosalicylic acid is a prodrug targeting dihydrofolate reductase in Mycobacterium tuberculosis. J. Biol. Chem. 2013, 288, 23447-23456. [CrossRef] [PubMed]

15. Marc, G.; Oniga, S.; Pirnau, A.; Duma, M.; Vlase, L.; Oniga, O. Rational Synthesis of Some New para-Aminobenzoic Acid Hybrids with Thiazolidin-2,4-diones with Antimicrobial Properties. ADMET and molecular docking evaluation. Rev. Chim. (Buchar.) 2019, 70, 769-775.

16. Scherman, M.S.; North, E.J.; Jones, V.; Hess, T.N.; Grzegorzewicz, A.E.; Kasagami, T.; Kim, I.H.; Merzlikin, O.; Lenaerts, A.J.; Lee, R.E.; et al. Screening a library of 1600 adamantyl ureas for anti-Mycobacterium tuberculosis activity in vitro and for better physical chemical properties for bioavailability. Bioorg. Med. Chem. 2012, 20, 3255-3262. [CrossRef] [PubMed]

17. Mureşan-Pop, M.; Kacsó, I.; Martin, F.; Simon, S.; Ştefan, R.; Bratu, I. Ambazone salt with p-aminobenzoic acid. J. Therm. Anal. Calorim. 2015, 120, 905-912. [CrossRef]

18. Farooq, M.; Al Marhoon, Z.M.; Taha, N.A.; Baabbad, A.A.; Al-Wadaan, M.A.; El-Faham, A. Synthesis of Novel Class of N-Alkyl-isatin-3-iminobenzoic Acid Derivatives and Their Biological Activity in Zebrafish Embryos and Human Cancer Cell Lines. Biol. Pharm. Bull. 2018, 41, 350-359. [CrossRef]

19. Krátký, M.; Vinšová, J.; Volková, M.; Buchta, V.; Trejtnar, F.; Stolaříková, J. Antimicrobial activity of sulfonamides containing 5-chloro-2-hydroxybenzaldehyde and 5-chloro-2-hydroxybenzoic acid scaffold. Eur. J. Med. Chem. 2012, 50, 433-440. [CrossRef]

20. Krátký, M.; Dzurková, M.; Janoušek, J.; Konečná, K.; Trejtnar, F.; Stolaříková, J.; Vinšová, J. Sulfadiazine Salicylaldehyde-Based Schiff Bases: Synthesis, Antimicrobial Activity and Cytotoxicity. Molecules 2017, 22, 1573. [CrossRef] 
21. Krátký, M.; Bősze, S.; Baranyai, Z.; Stolaříková, J.; Vinšová, J. Synthesis and biological evolution of hydrazones derived from 4-(trifluoromethyl)benzohydrazide. Bioorg. Med. Chem. Lett. 2017, 27, 5185-5189. [CrossRef] [PubMed]

22. Krátký, M.; Vinšová, J.; Stolaříková, J. Antimicrobial activity of rhodanine-3-acetic acid derivatives. Bioorg. Med. Chem. 2017, 25, 1839-1845. [CrossRef]

23. Shrivastava, S.K.; Srivastava, P.; Upendra, T.V.R.; Tripathi, P.N.; Sinha, S.K. Design, synthesis and evaluation of some $N$-methylenebenzenamine derivatives as selective acetylcholinesterase (AChE) inhibitor and antioxidant to enhance learning and memory. Bioorg. Med. Chem. 2017, 25, 1471-1480. [CrossRef] [PubMed]

24. Shetty, M.M.; Sadanandam, Y.S.; Sattur, P.B. Synthese und Pharmakologie von substituierten N-Benzyliden-Derivaten. Arch. Pharm. 1979, 312, 721-726. [CrossRef] [PubMed]

25. Brewster, C.M.; Millam, L.H. Phototropic and Thermotropic Anils from 5-Bromosalicylaldehyde. J. Am. Chem. Soc. 1933, 55, 763-766. [CrossRef]

26. Mayadeo, M.S.; Dhakappa, V.P. Determination of stability constants of $\mathrm{N}$-[2-hydroxy-5-methylbenzylidene]-4-carboxyaniline complexes with $\mathrm{Y}^{3+}, \mathrm{La}^{3+}, \mathrm{Pr}^{3+}, \mathrm{Nd}^{3+}, \mathrm{Sm}^{3+}, \mathrm{Gd}^{3+}$ and $\mathrm{Dy}^{3+}$. J. Indian Chem. Soc. 1981, 58, 1010.

27. Nasr, G.; Cristian, A.; Barboiu, M.; Vullo, D.; Winum, J.-Y.; Supuran, C.T. Carbonic anhydrase inhibitors. Inhibition of human cytosolic isoforms I and II with (reduced) Schiff's bases incorporating sulfonamide, carboxylate and carboxymethyl moieties. Bioorg. Med. Chem. 2014, 22, 2867-2874. [CrossRef]

28. UNIVERSITÀ DEGLI STUDI DI SALERNO; Longo, P.; Saturnino, C.; Arra, C.; Palma, G.; Mariconda, A.; Sinicropi, M.S.; Puoci, F.; Iacopetta, D. Hydroxybenzene derivatives having a N-aryl substitute imino group and use thereof in the treatment of solid tumours. Patent WO2018/116158; A1, 28 June 2018.

29. Cronenberger, L.; Gaige, T.; Pacheco, H.; Pillon, D. Nouvelles bases de Schiff dérivées des aldehydes dihalogéno-3,5 salicyliques et possédant des propriétés antifongiques et antibactériennes. Chim. Ther. 1968, 3, 87-99.

30. Ujiie, T. Experimental Anticancer Studies. XXVIII. Anticancer Activity of Some Nitrofuran Derivatives. Chem. Pharm. Bull. 1966, 14, 461-466. [CrossRef]

31. Silku, P.; Özkinali, S.; Öztürk, Z.; Asan, A.; Köse, D.A. Synthesis of novel Schiff Bases containing acryloyl moiety and the investigation of spectroscopic and electrochemical properties. J. Mol. Struct. 2016, 1116, 72-83. [CrossRef]

32. Bhunia, M.; Hota, P.K.; Vijaykumar, G.; Adhikari, D.; Mandal, S.K. A Highly Efficient Base-Metal Catalyst: Chemoselective Reduction of Imines to Amines Using An Abnormal-NHC-Fe(0) Complex. Organometallics 2016, 35, 2930-2937. [CrossRef]

33. EUCAST DISCUSSION DOCUMENT. E.Dis 5.1. Determination of minimum inhibitory concentrations (MICs) of antibacterial agents by broth dilution. Clin. Microbiol. Infect. 2003, 9, 1-7.

34. Krátký, M.; Vinšová, J.; Novotná, E.; Mandíková, J.; Trejtnar, F.; Stolař́ková, J. Antibacterial Activity of Salicylanilide 4-(Trifluoromethyl)benzoates. Molecules 2013, 18, 3674-3688. [CrossRef] [PubMed]

35. EUCAST DEFINITIVE DOCUMENT EDEF 7.3.1. Method for the Determination of Broth Dilution Minimum Inhibitory Concentrations of Antifungal Agents for Yeasts. 2017, pp. 1-21. Available online: http://www.eucast.org/fileadmin/src/media/PDFs/EUCAST_files/AFST/Files/EUCAST_E_Def_7_ 3_1_Yeast_testing_definitive.pdf (accessed on 20 October 2019).

36. EUCAST DEFINITIVE DOCUMENT E.DEF 9.3.1. Method for the Determination of Broth Dilution Minimum Inhibitory Concentrations of Antifungal Agents for Conidia Forming Moulds. 2017, pp. 1-23. Available online: http://www.eucast.org/fileadmin/src/media/PDFs/EUCAST_files/AFST/Files/EUCAST_E_Def_9_3_ 1_Mould_testing_definitive.pdf (accessed on 20 October 2019).

37. Ordonez, A.A.; Weinstein, E.A.; Bambarger, L.E.; Saini, V.; Chang, Y.S.; DeMarco, V.P.; Klunk, M.H.; Urbanowski, M.E.; Moulton, K.L.; Murawski, A.M.; et al. A Systematic Approach for Developing Bacteria-Specific Imaging Tracers. J. Nucl. Med. 2017, 58, 144-150. [CrossRef]

38. Elsaman, T.; Mohamed, M.S.; Mohamed, M.A. Current development of 5-nitrofuran-2-yl derivatives as antitubercular agents. Bioorg. Chem. 2019, 88, 102969. [CrossRef]

39. Laborda, P.; Zhao, Y.; Ling, J.; Hou, R.; Liu, F. Production of Antifungal p-Aminobenzoic Acid in Lysobacter antibioticus OH13. J. Agric. Food Chem. 2018, 66, 630-636. [CrossRef]

40. Meir, Z.; Osherov, N. Vitamin Biosynthesis as an Antifungal Target. J. Fungi 2018, 4, 72. [CrossRef] 
41. Iliev, I.; Kontrec, D.; Detcheva, R.; Georgieva, M.; Balacheva, A.; Galić, N.; Pajpanova, T. Cancer cell growth inhibition by aroylhydrazone derivatives. Biotechnol. Biotec. Eq. 2019, 33, 756-763. [CrossRef]

42. Gerets, H.H.J.; Tilmant, K.; Gerin, B.; Chanteux, H.; Depelchin, B.O.; Dhalluin, S.; Atienzar, F.A. Characterization of primary human hepatocytes, HepG2 cells, and HepaRG cells at the mRNA level and CYP activity in response to inducers and their predictivity for the detection of human hepatotoxins. Cell Biol. Toxicol. 2012, 28, 69-87. [CrossRef]

43. Kamalian, L.; Chadwick, A.E.; Bayliss, M.; French, N.S.; Monshouwer, M.; Snoeys, J.; Park, B.K. The utility of HepG2 cells to identify direct mitochondrial dysfunction in the absence of cell death. Toxicol. In Vitro 2015, 29, 732-740. [CrossRef]

44. Hradilová, L.; Poláková, M.; Dvořáková, B.; Hajdúch, M.; Petruš, L. Synthesis and cytotoxicity of some D-mannose click conjugates with aminobenzoic acid derivatives. Carbohydr. Res. 2012, 361, 1-6. [CrossRef] [PubMed]

(C) 2019 by the authors. Licensee MDPI, Basel, Switzerland. This article is an open access article distributed under the terms and conditions of the Creative Commons Attribution (CC BY) license (http://creativecommons.org/licenses/by/4.0/). 